\title{
Immediate Implant Placement in Non-Infected Sockets versus Infected Sockets: a Systematic Review and Meta-Analysis
}

\author{
Aza Saijeva1, Gintaras Juodzbalys ${ }^{1,2}$ \\ ${ }^{1}$ Department of Oral and Maxillofacial Surgery, Lithuanian University of Health Sciences, Lithuania. \\ ${ }^{2}$ Dental Implant Centre "Stilus Optimus", Kaunas, Lithuania.
}

\author{
Corresponding Author: \\ Aza Saijeva \\ Sukileliu pr. 53, 50105, Kaunas \\ Lithuania \\ Phone: +37068433814 \\ E-mail: azasaijeva@hotmail.com
}

\begin{abstract}
Objectives: The aim of this systematic review is to compare immediate implant placement in infected extraction sockets with non-infected extraction sockets in terms of implant survival and function.

Material and Methods: An electronic search was conducted in PubMed, ScienceDirect, ISI Web of Knowledge and Google Scholar between January 2010 and February 2020. Studies evaluating implant survival rate and main clinical parameters were included for a qualitative and quantitative analysis.

Results: In total, nine studies were included and a pool of 2281 sockets were analysed. Compared with the non-infected group, the infected group showed no significant differences in implant survival rates (risk ratio $[\mathrm{RR}]=0.99 ; 95 \%$ confidence interval $[\mathrm{CI}]=0.98$ to $1 ; \mathrm{P}=0.08$ ). No significant statistical differences were found in marginal bone level (mean difference $[\mathrm{MD}]$ $=-0.03 ; 95 \% \mathrm{CI}=-0.1$ to $0.04 ; \mathrm{P}=0.41)$, marginal gingival level $(\mathrm{MD}=-0.07 ; 95 \% \mathrm{CI}=-0.17$ to $0.04 ; \mathrm{P}=0.23)$, probing depth $(\mathrm{MD}=0.06 ; 95 \% \mathrm{CI}=-0.24$ to $0.36 ; \mathrm{P}=0.7)$, modified bleeding index $(\mathrm{MD}=-0.00162196 ; 95 \% \mathrm{CI}=-0.09$ to 0.09 ; $\mathrm{P}=0.97)$ and slight but significant changes were seen in width of keratinized gingiva $(\mathrm{MD}=0.25 ; 95 \% \mathrm{CI}=-0.3$ to $0.8 ; \mathrm{P}=$ 0.38 ) between the groups at the latest follow-up.

Conclusions: There were no significant difference in implant survival rates, marginal bone level, marginal gingival level, modified bleeding index and probing depth between infected sockets and non-infected sockets. However, slight but significant changes were seen in width of keratinized gingiva favouring the non-infected group.
\end{abstract}

Keywords: dental implantation; dental implants; infection; periapical granuloma; tooth socket.

Accepted for publication: 30 June 2020

To cite this article:

Saijeva A, Juodzbalys G.

Immediate Implant Placement in Non-Infected Sockets versus Infected Sockets: a Systematic Review and Meta-Analysis

J Oral Maxillofac Res 2020;11(2):e1

URL: http://www.ejomr.org/JOMR/archives/2020/2/e1/v11n2e1.pdf

doi: $10.5037 /$ jomr.2020.11201 


\section{INTRODUCTION}

Currently, the treatment of choice to replace missing teeth has been implant supported dental rehabilitation due to its success rate and good long-term prognosis [1]. The first endosteal titanium implant was placed successfully in 1965 by Brånemark [2]. During the 1980s Brånemark introduced the original protocol for implant therapy and the recommendation included post extraction healing time of 5 - 6 months before the implant was placed into the alveolar ridge $[\underline{1}, \underline{2}]$. The conventional protocol was established on the belief that only complete hard and soft tissue healing would guarantee a favourable osseointegration [ $\underline{3}$ ]. The immediate implant placement in fresh-socket was firstly introduced in 1976 and in the year 1989 the first immediate implant was placed [2] . Due to the modern implantology and its findings of new designs and surfaces, it is now possible to modify the classical protocol that was introduced decades ago []‥ As of current, there are four different methods regarding the placement of implants into edentulous sites [ $[\underline{6}]$ :

- Immediate implant placement, when the implant is placed directly after the extraction;

- Early implant placement, the implant is placed 1 - 2 months after the extraction;

- Delayed implant placement, the implant is placed 3 - 4 months after the extraction;

- Late implant placement, when the implant is placed more than 4 months after the tooth extraction.

Immediate implant placement in fresh-socket is a protocol that has received a lot of attention and is now considered a common treatment step with predictable and successful results $[\underline{2}, \underline{7}, \underline{8}]$. Immediate post extraction implant placement offers advantages such as:

- Reduced number of surgical interventions and shortening of the treatment procedure, ultimately leading to an increased patient satisfaction [2-6, 8-11];

- Optimal soft tissue aesthetics due to the preservation of soft tissue envelope $[2, \underline{4}, \underline{6}]$.

However, immediate implant placement does not always provide optimal clinical outcomes. Preclinical and human studies documented suggest that this surgical protocol may not preserve the buccal bone crest. To prevent the dimensional changes of the alveolar bone and the soft tissue during immediate implant placement, numerous surgical techniques have been suggested $[\underline{1}, \underline{12}]$ :

- Flapless technique;

- Use of bone grafts;

- Use of connective tissue grafts;
- Provisional restorations;

- Highlighting the importance of buccal bone plate thickness;

- Importance of alveolar bone thickness.

A recent clinical trial shows that using a bone replacement graft between the implant and the buccal bone plate notably improves the preservation of the bone after immediate implant placement [12].

A non-infected extraction socket has great benefit to the survival rate of immediate implant placement. However, in practice, teeth extractions are largely due to presence of chronic pathology that later leads to endodontic or periodontal apical lesions $[13,14]$. Thus, the aim of this systematic review was to evaluate if immediate implant placement in infected extraction sockets can be considered as successful in comparison to non-infected sockets.

\section{MATERIAL AND METHODS Protocol}

The reporting of this systematic review was conducted by following the Preferred Reporting Items for Systematic Reviews and Meta-Analyses (PRISMA) [15].

\section{Focus question}

The following focus question was framed according to the problem, intervention, comparison and outcome (PICO) process (Table 1):

What is the outcome of immediate implant placement in patients with infected sockets versus non-infected sockets with the evaluation of survival rate, probing depth, marginal bone level, marginal gingival level, modified bleeding index and width of keratinized gingiva?

Table 1. PICO framework of the framed clinical question

\begin{tabular}{l|l}
\hline \multicolumn{1}{c|}{ Definition } & \multicolumn{1}{c}{ Description } \\
\hline Patient (P) & Patients with infected sockets \\
\hline Intervention (I) & Immediate implant placement \\
\hline Comparison (C) & A control group with non-infected sockets \\
\hline Outcome (O) & $\begin{array}{l}\text { Implant success by evaluating: survival rate, } \\
\text { marginal bone level, marginal gingival level, } \\
\text { width of keratinized gingiva, modified } \\
\text { bleeding index and probing depth. }\end{array}$ \\
\hline Focus question & $\begin{array}{l}\text { What is the outcome of immediate implant } \\
\text { placement in patients with infected sockets } \\
\text { versus non-infected sockets with the evaluation } \\
\text { of survival rate, marginal bone level, marginal } \\
\text { gingival level, width of keratinized gingiva, } \\
\text { modified bleeding index and probing depth? }\end{array}$ \\
\hline
\end{tabular}




\section{Information sources}

An electronic search for articles in English language was performed using PubMed, ISI Web of Science, ScienceDirect and Google Scholar from January 2010 to February 2020.

\section{Search strategy}

The literature search strategy was done by following the PRISMA guidelines using PubMed, ISI Web of Science, ScienceDirect electronic databases and Google Scholar. The search was conducted using a combination of different search terms (Table 2).

\section{Types of publications}

The systematic review included only English clinical studies done on humans. Publications that were lacking full text, in vitro studies and studies done on animals were excluded.

\section{Types of studies}

The systematic review included all human retrospective and prospective observational studies published from January 2010 to February 2020.

\section{Types of participants/population}

Subjects, whose extraction sockets were classified as having infection and that were treated with immediate implant placement, were included in this systematic review.

\section{Outcome variables}

The primary outcome variable was the implant survival rates. The secondary outcome variables were the mean changes in marginal bone level (MBL), marginal gingival level (MGL), width of keratinized gingiva (WKG), modified bleeding index (mBI) and probing depth (PD).

\section{Inclusion criteria}

Studies were included if they followed the applied criteria:

- Studies with a sample size of $>5$ patients in each group;

- Minimum follow-up of 6 months;

- Evaluated with one of the outcomes;

- If the sockets were classified as having an infection.

\section{Exclusion criteria}

Studies were excluded if they met any of the following applied criteria:

- Clinical studies with no control group;

- Animal studies;

- Non-English articles;

- Studies that did not mention the socket morphology;

- No clear methodology description;

- Secondary sources.

\section{Data extraction}

The following data was extracted from the articles included in this review:

- First author and publication year;

- Study design;

- Total number of patients;

- Total number of sockets and type of socket pathology;

- Follow-up period;

- Implant system;

- Site of implant placement;

- Number of smoking patients;

- Treatment methodology including flap technique, granulation tissue removal, bone graft, loading time, mouth rinse and antibiotic prophylaxis;

- Implant failure and implant survival outcomes;

- Secondary outcome measures namely MBL, MGL, WKG, $\mathrm{mBI}$ and PD.

Table 2. Keywords used to conduct the literature search

\begin{tabular}{c|l}
\hline Concept & \multicolumn{1}{c}{ Keywords } \\
\hline First keyword terms & $\begin{array}{l}\text { "Infected socket*" OR "Periapical lesion*” OR "Endodontic lesion*" OR "Periodontal lesion*” } \\
\text { OR "Radicular lesion*" OR "Periradicular lesion*” OR "Apical lesion*” OR "Apical pathology" } \\
\text { OR "Periradicular pathology" OR "Radicular pathology" OR "Endodontic pathology" OR } \\
\text { "Periapical pathology" OR "Apical pathological feature*” OR "Apical periodontitis" }\end{array}$ \\
\hline Second keyword terms & "Immediate implant*" OR "Fresh-socket*" OR "Fresh extraction*” OR "Post-extraction" \\
\hline
\end{tabular}

First keyword terms and second keyword terms were combined with AND.

*truncation symbol. 


\section{Statistical analysis}

The meta-analysis was conducted in Review Manager Software version 5.3 (The Cochrane Collaboration, Oxford, UK). The Higgins index $\left(\mathrm{I}^{2}\right)$ statistic test was used to measure the heterogeneity across the studies. Cochrane Handbook guidelines were adopted to interpret the heterogeneity with 0 to $40 \%$ representing low, 30 to $60 \%$ may represent moderate heterogeneity, and 50 to $60 \%$ may represent substantial heterogeneity and 75 to $100 \%$ representing considerable heterogeneity [16]. The level of P-value was set at $<0.05$.

The Mantel-Haenszel method was used for implant survival rates and implant failure rates (dichotomous outcome variables) together with fixed-effects model. The effect size between the control group and the test group was expressed as risk ratios (RR) and 95\% confidence intervals (CIs). If significant heterogeneity $(>75 \%)$ was seen, the random-effects model was chosen.

The same process was followed for the continuous outcome variables (MBL, MGL, WKG, $\mathrm{mBI}$ and $\mathrm{PD}$ ). However, they were based on inverse variance (IV) with effect size expressed as mean difference (MD) in millimetres and $95 \%$ CIs. A funnel plot was made with software (Review Manager Version 5.3; The Cochrane Collaboration, Oxford, UK) for the primary outcome, to investigate the possibility of publication bias.

\section{Risk of bias assessment}

The risk of bias assessment was performed using the Joanna Briggs Institute Critical Appraisal Checklist for Cohort Studies [17]. Questions that were evaluated can be found in Table 3 .

\section{RESULTS \\ Study selection}

A total of 316 publications were screened, from which 294 articles were excluded based on the titles. In the next step the abstracts of all the 22 remaining studies were assessed for eligibility based on inclusion criteria. If an abstract provided insufficient amount of information to decide whether or not to include the article, the full version of the article was downloaded for further detailed evaluation. Subsequently, the full-text of the articles that were potentially relevant was obtained for assessment of the eligibility. A total of 12 full-articles were reviewed for inclusion and exclusion criteria in order to make the final decision.

After a detailed review, nine records met all the required criteria and were included in this review [1826]. Figure 1 shows the PRISMA flow diagram which demonstrates the number of publications identified, screened, assessed for eligibility and included in this review.

\section{Study exclusion}

Three studies were excluded after a full-text review due to: lack of control group [27], publication of the same patient records [28] and lack of control and test group details [르].

\section{Quality assessment of the included studies}

The quality assessment of all the cohort studies revealed moderate or good qualities; the scoring of each study is summarized in Table 4.

Table 3. Joanna Briggs Institute Critical Appraisal Checklist for Cohort Studies

\begin{tabular}{c|l}
\hline $\begin{array}{c}\text { Question } \\
\text { number }\end{array}$ & \multicolumn{1}{c}{ Defined question } \\
\hline Q1 & Were the two groups similar and recruited from the same population? \\
\hline Q2 & Were the exposures measured similarly to assign people to both exposed and unexposed groups? \\
\hline Q3 & Was the exposure measured in a valid and reliable way? \\
\hline Q4 & Were confounding factors identified? \\
\hline Q5 & Were strategies to deal with confounding factors stated? \\
\hline Q6 & Were the groups/participants free of the outcome at the start of the study (or at the moment of exposure)? \\
\hline Q7 & Were the outcomes measured in a valid and reliable way? \\
\hline Q8 & Was the follow-up time reported and sufficient to be long enough for outcomes to occur? \\
\hline Q9 & Was follow-up complete, and if not, were the reasons to loss to follow-up described and explored? \\
\hline Q10 & Were strategies to address incomplete follow up utilized? \\
\hline Q11 & Was appropriate statistical analysis used? \\
\hline
\end{tabular}




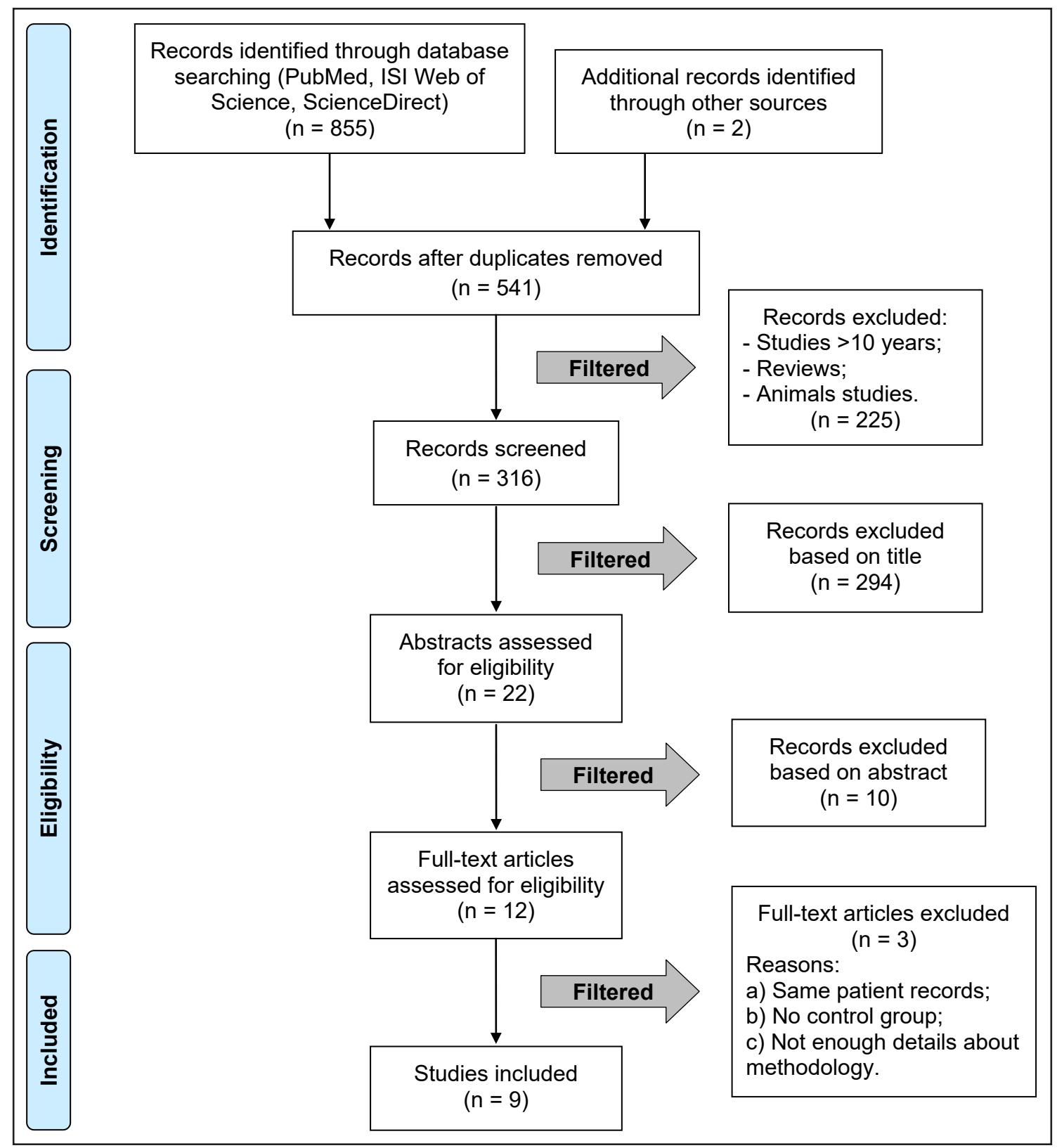

Figure 1. PRISMA flow diagram demonstrating the study selection.

Table 4. Quality assessment of all the included cohort studies using the Joanna Briggs Institute Critical Appraisal Checklist for Cohort Studies

\begin{tabular}{|c|c|c|c|c|c|c|c|c|c|c|c|c|c|}
\hline \multirow{2}{*}{ Study } & \multirow{2}{*}{$\begin{array}{c}\text { Year of } \\
\text { publication }\end{array}$} & \multirow{2}{*}{$\begin{array}{l}\text { Study } \\
\text { design }\end{array}$} & \multicolumn{11}{|c|}{ Checklist } \\
\hline & & & Q1 & Q2 & Q3 & Q4 & Q5 & Q6 & Q7 & Q8 & Q9 & Q10 & Q11 \\
\hline Montoya-Salazar et al. [18] & 2014 & Prospective & $?$ & + & + & - & - & + & + & + & + & N/A & + \\
\hline Jung et al. [19] & 2012 & Prospective & + & + & + & + & N/A & + & + & + & + & N/A & + \\
\hline Crespi et al. [20] & 2010 & Prospective & + & + & + & N/A & $\mathrm{N} / \mathrm{A}$ & + & + & + & + & N/A & + \\
\hline Crespi et al. [21] & 2010 & Prospective & + & + & + & N/A & N/A & + & + & + & + & N/A & + \\
\hline Hita-Iglesias et al. [22] & 2016 & Prospective & + & + & + & N/A & $\mathrm{N} / \mathrm{A}$ & + & + & + & + & N/A & + \\
\hline Blus et al. [23] & 2015 & Prospective & $?$ & + & + & - & - & + & + & + & + & $\mathrm{N} / \mathrm{A}$ & + \\
\hline Bell et al. [24] & 2011 & Retrospective & + & + & + & + & + & + & + & + & + & N/A & + \\
\hline Fugazzotto [25] & 2012 & Retrospective & + & + & + & N/A & $\mathrm{N} / \mathrm{A}$ & + & + & + & + & N/A & + \\
\hline Zuffetti et al. [26] & 2017 & Retrospective & $?$ & + & + & + & - & + & + & + & + & $\mathrm{N} / \mathrm{A}$ & + \\
\hline
\end{tabular}

N/A = not applicable; $?=$ unclear; $+=$ yes; $-=$ no. 


\section{Study characteristics}

The characteristics and details of the treatment procedures of the included studies are presented in Table 5 and 6 . Three of the included clinical trials were of retrospective design [24-26] and the other remaining six were of prospective design [18-23]. In total, a pool of 1346 patients and 2281 sockets were used in this present systematic review. A total of 933 immediate implants were placed in infected sites and 1348 in non-infected sites. Seven of the studies included smoking patients $[18-21,24-26]$. Only two of them specified the total number of smokers $[19,24]$. One of the studies did not mention if smoking patients were included or excluded [23]. Three of the articles mentioned the exclusion of heavy smokers $(>10$ cigarettes a day) $[\underline{20}, \underline{21}, \underline{25}]$.

Most of the studies indicated that implants has been placed in the incisor, canine or premolar area [18-23], while two of the clinical trials included the molar area as well $[\underline{24}, \underline{26}]$. Only one study exclusively reported on the incisor replacement [25]. Six of the studies used flapless approach [20-25], while two of them proceeded with a flap technique $[\underline{18}, 19]$ and only one used both flap and flapless approach [26]. No grafting material was used in three of the studies reviewed [20-22]. Four of the included studies used xenograft materials $[18,19,23,26]$, one used both autograft or xenograft together with platelet rich plasma [24], whiles the remaining one clinical trial included autograft, allograft or xenograft [25].

A delayed loading protocol was followed for all implants in six of the studies $[\underline{18}, \underline{19}, \underline{21}, \underline{22}, \underline{24}, \underline{25}]$. Only one study used an immediate loading protocol [20], whiles the two remaining used different types of loading protocols $[23,26]$. The patients received a preoperative antibiotic prophylaxis in seven of the studies $[\underline{18}, \underline{20-24}, \underline{26}]$ and five of the studies reported about prescription of postoperative antibiotics for the patients [18-22,25-26]. A postoperative instruction on chlorhexidine rinse was made in five of the clinical trials $[\underline{18-21}, \underline{26}]$, while only one study followed a preoperative mouth rinse protocol [26].

All of the included studies reported the number of failed implants [18-26] while four of them reported about MBL changes [18-21]. Changes in mBI were reported by three clinical trials $[\underline{18}, \underline{20}, \underline{21}]$, MGL and PD by two $[18,21]$ and WKG changes by three $[18,19,21]$. The minimum follow-up period of the secondary outcome variables (MBL, MGL, PD, mBI and $\mathrm{WKG}$ ) was one year and the maximum followup period was five years. Table 7 shows detailed information about the extracted data from the different follow-up periods regarding MBL, MGL, PD,
$\mathrm{mBI}$ and $\mathrm{WKG}$.

\section{Quantitative synthesis \\ Implant survival rates and failures (primary outcome variables)}

In total 933 immediate implants were placed in infected sockets and 1348 in non-infected sockets [18-26]. The number of failed implants was 22 for the infected socket group and 19 for the noninfected socket group resulting in overall implant survival rates of $97.64 \%(911 / 933)$ for the infected group and $98.57 \%(1329 / 1348)$ for the non-infected group (Figure 2). Both of the groups showed similar results ( $\mathrm{RR}=0.99 ; 95 \% \mathrm{CI}=0.98$ to $1 ; \mathrm{P}=0.08$ ). Additionally, there was no significant difference in the implant failure rates between the infected and noninfected groups $(\mathrm{RR}=1.8 ; 95 \% \mathrm{CI}=0.98$ to $3.31 ; \mathrm{P}=$ $0.06)$ as seen in Figure 3.

\section{Marginal bone level changes}

Four of the included clinical trials analyzed the MBL measurements [18-21]. In total 242 implants in the infected sockets and 126 implants in non-infected sockets were included in this analysis (Figure 4A - C). One of the studies reported three follow-up periods of 1,2 and 3 years [ [18]. Another study reported three follow-up periods but of 1, 2 and 4 years [20]. The last two studies reported 1 and 2 years [21] and 5 years [19] respectively. No significant difference was found between the different groups at follow-up times, at 1 year the MD was $-0.05(95 \% \mathrm{CI}=-0.15$ to 0.04 ; $\mathrm{P}=0.25)$ at year 2 the $\mathrm{MD}$ was $0.12(95 \% \mathrm{CI}=-0.14$ to $0.38 ; \mathrm{P}=0.36)$ and at year 3 or more the MD was $-0.03(95 \% \mathrm{CI}=-0.1$ to $0.04 ; \mathrm{P}=0.41)$.

\section{Marginal gingival level changes}

Two of the included clinical trials analyzed the MGL changes $[\underline{18}, \underline{21}]$. The total number of implants included was 33 in the infected group and 33 in the non-infected group (Figure 5A and 5B). Meta-analysis showed no significant difference between the two groups. At 1 year follow-up the MD was $-0.06(95 \%$ $\mathrm{CI}=-0.15$ to $0.03 ; \mathrm{P}=0.17)$ and at 2 year follow-up the $\mathrm{MD}$ was $-0.07(95 \% \mathrm{CI}=-0.17$ to $0.04 ; \mathrm{P}=0.23)$.

\section{Probing depth changes}

Two of the included clinical trials analyzed the peri-implant $\mathrm{PD}$ measurements $[\underline{18}, \underline{21}]$. In total, 33 implants were included in both infected and non-infected groups (Figure 6A and 6B). 
Table 5. Characteristics of the included studies

\begin{tabular}{|c|c|c|c|c|c|c|c|c|}
\hline Study & $\begin{array}{l}\text { Patients } \\
\text { (n) }\end{array}$ & $\begin{array}{c}\text { Sockets } \\
\text { (n) }\end{array}$ & Smoking patients & IS pathology & $\begin{array}{c}\text { Age } \\
\text { (years) }\end{array}$ & Site & $\begin{array}{l}\text { Follow-up } \\
\text { (months) }\end{array}$ & Implant system \\
\hline Montoya-Salazar et al. [18] & 18 & 36 (IS:18, NIS:18 & Included & Chronic periapical lesion & $18-50$ & Incisors, canines and premolars & 36 & MIS Cl implants (MIS Implants Technologies Ltd.; Tel Aviv, Israel) \\
\hline Jung et al. [19] & 27 & 27 (IS:12, NIS:15) & Included & Periapical pathologies & $31-87$ (IS) $28-82$ (NIS) & Incisors, canines and premolars & 60 & Straumann ${ }^{\otimes}$ Standard Plus or Tapered Effect (Straumann AG; Basal, Switzerland) \\
\hline Crespi et al. [20] & 37 & 275 (IS:197, NIS:78) & $\begin{array}{l}\text { Heavy smokers excluded } \\
\text { (>10 cigarettes/day })\end{array}$ & Chronic periodontal lesions & $32-71$ & Incisors, canines and premolars & 48 & Sweden and Martina SPA, Due Carrare, Padova, Italy \\
\hline Crespi et al. [21] & 30 & 30 (IS:15, NIS:15) & $\begin{array}{l}\text { Heavy smokers excluded } \\
(>10 \text { cigarettes/day) }\end{array}$ & $\begin{array}{l}\text { Periapical lesions and } \\
\text { radiolucencies } \\
\end{array}$ & $34-71$ & Incisors, canines and premolars & 24 & Seven (Sweden and Martina SPA, Due Carrare, Padova, Italy) \\
\hline Hita-Iglesias et al. [22] & 60 & 168 (IS:66, NIS:102) & Non-smokers only & Chronic periapical lesions & $18-72$ & Incisors, canines and premolars & 12 & Zimmer dental, USA \\
\hline Blus et al. [23] & 86 & 168 (IS:83, NIS:85) & No data & Acute and chronic infection & $26-77$ & Incisors, canines and premolars & 12 & Leader Implants; Milan, Italy or and Bioner Sistemas Implantológicos, Barcelona, Spain \\
\hline Bell et al. [24] & 655 & 922 (IS:285, NIS:637) & Included & Chronic periapical lesion & Mean: $58.4 \mathrm{IS} ; 60.1 \mathrm{NIS}$ & Incisors, canines, premolars and molars & $3-93$ & Straumann ${ }^{\mathbb{1}}$ Tissue Level or Bone Level SLA (Straumann AG; Basal, Switzerland) \\
\hline Fugazzotto [25] & 64 & 128 (IS:64, NIS:64) & $\begin{array}{c}\text { Heavy smokers excluded } \\
(>10 \text { cigarettes/day })\end{array}$ & Periapical pathologies & $21-71$ & ( & $24-117$ & 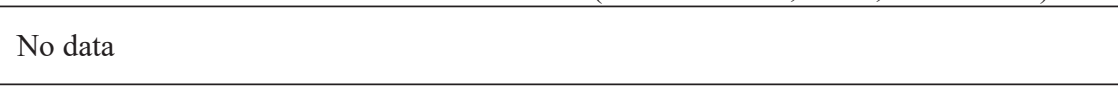 \\
\hline
\end{tabular}

$\mathrm{n}=$ numbers; IS = infected socket; NIS = non-infected socket; ASA = The American Society of Anaesthesiologists physical status classification system.

Table 6. Details of the treatment procedures of the included studies

\begin{tabular}{|c|c|c|c|c|c|c|c|c|}
\hline Study & $\begin{array}{c}\text { Flap } \\
\text { technique }\end{array}$ & $\begin{array}{c}\text { Granulation } \\
\text { tissue }\end{array}$ & Bone graft & Loading time & $\begin{array}{c}\text { Preoperative } \\
\text { antibiotic prophylaxis }\end{array}$ & $\begin{array}{c}\text { Preoperative } \\
\text { chlorhexidine rinse }\end{array}$ & \begin{tabular}{|c|} 
Postoperative \\
antibiotic prophylaxis
\end{tabular} & $\begin{array}{c}\text { Postoperative } \\
\text { chlorhexidine rinse }\end{array}$ \\
\hline Montoya-Salazar et al. [18] & Flap & Removed & Xenograft & Delayed & Yes & No & Yes & Yes \\
\hline Jung et al. [19] & Flap & Removed & Xenograft & Delayed & No & No & Yes & Yes \\
\hline Crespi et al. [20] & Flapless & Removed & None & Immediate & Yes & No & Yes & Yes \\
\hline Crespi et al. [21] & Flapless & Removed & None & Delayed & Yes & No & Yes & Yes \\
\hline Hita-Iglesias et al. [22] & Flapless & Removed & None & Delayed & Yes & No & Yes & No \\
\hline Blus et al. [23] & Flapless & Removed & Xenograft & Immediate, early and delayed & Yes & No & No & No \\
\hline Bell et al. [24] & Flapless & Removed & $\begin{array}{c}\text { Autograft and/or xenograft } \\
\text { together with platelet rich plasma }\end{array}$ & Delayed & Yes & Yes & No & No \\
\hline Fugazzotto [25] & Flapless & Removed & Autograft or allograft or xenograft & Delayed & No & No & Yes & No \\
\hline Zuffetti et al. [26] & Flapless or flap & Removed & Xenograft & Immediate, early and delayed & Yes & No & Yes & Yes \\
\hline
\end{tabular}

Table 7. Data of the primary and secondary outcomes of the included studies

\begin{tabular}{|c|c|c|c|c|c|c|c|c|c|c|c|c|c|c|c|c|c|c|}
\hline \multirow{3}{*}{ Study } & \multirow{2}{*}{\multicolumn{3}{|c|}{$\begin{array}{c}\text { Number of } \\
\text { implants } \\
\text { (n) }\end{array}$}} & \multirow{2}{*}{\multicolumn{3}{|c|}{$\begin{array}{l}\text { Failed implants } \\
\text { (n) }\end{array}$}} & \multirow{2}{*}{\multicolumn{2}{|c|}{$\begin{array}{l}\text { Implant } \\
\text { survival rate } \\
(\%)\end{array}$}} & \multirow{2}{*}{\multicolumn{2}{|c|}{$\begin{array}{c}\begin{array}{c}\text { MBL } \\
(\mathrm{mm})\end{array} \\
\text { Mean (SD) }\end{array}$}} & \multirow{2}{*}{\multicolumn{2}{|c|}{$\begin{array}{c}\begin{array}{c}\text { MGL } \\
(\mathrm{mm})\end{array} \\
\text { Mean (SD) }\end{array}$}} & \multirow{2}{*}{\multicolumn{2}{|c|}{$\begin{array}{c}\begin{array}{c}\text { PD } \\
(\mathrm{mm})\end{array} \\
\text { Mean (SD) }\end{array}$}} & \multirow{2}{*}{\multicolumn{2}{|c|}{$\begin{array}{c}\begin{array}{c}\mathrm{mBI} \\
(\mathrm{mm})\end{array} \\
\text { Mean (SD) }\end{array}$}} & \multirow{2}{*}{\multicolumn{2}{|c|}{$\begin{array}{c}\begin{array}{c}\text { WKG } \\
(\mathrm{mm})\end{array} \\
\text { Mean (SD) }\end{array}$}} \\
\hline & & & & & & & & & & & & & & & & & & \\
\hline & IS & NIS & Total & IS & NIS & Total & IS & NIS & \begin{tabular}{|c|} 
IS \\
\end{tabular} & NIS & IS & \begin{tabular}{|c|} 
NIS \\
\end{tabular} & IS & \begin{tabular}{|c|} 
NIS \\
\end{tabular} & \begin{tabular}{|c|} 
IS \\
\end{tabular} & \begin{tabular}{|c|} 
NIS \\
\end{tabular} & IS & NIS \\
\hline $\begin{array}{l}\text { Montoya-Salazar et } \\
\text { al. [18] }\end{array}$ & 18 & 18 & 36 & 1 & 0 & 1 & 94.4 & 100 & \begin{tabular}{|l|} 
1 years: $0.73(0.22) ;$ \\
2 years: $0.84(0.15) ;$ \\
3 years: $0.53(0.13)$
\end{tabular} & $\begin{array}{l}1 \text { years: } 0.73(0.29) ; \\
2 \text { years: } 0.54(0.15) ; \\
3 \text { years: } 0.60(0.16)\end{array}$ & $\begin{array}{l}1 \text { years: } 0.88(0.75) ; \\
2 \text { years: } 0.83(0.85) ; \\
3 \text { years: } 1(0.59)\end{array}$ & $; \begin{array}{l}1 \text { years: } 1.13(0.23) ; \\
2 \text { years: } 1.11(0.21) ; \\
3 \text { years: } 1.16(0.24)\end{array}$ & $\begin{array}{l}1 \text { years: } 2.53(0.44) ; \\
2 \text { years: } 2.76(0.8) ; \\
3 \text { years: } 2.51(0.44)\end{array}$ & $\begin{array}{l}1 \text { years: } 2.44(0.28) ; \\
2 \text { years: } 2.6(0.37) ; \\
3 \text { years: } 2.53(0.44)\end{array}$ & \begin{tabular}{|l|}
1 years: $0.88(0.75) ;$ \\
2 years: $0.83(0.85) ;$ \\
3 years: $0.94(0.63)$
\end{tabular} & \begin{tabular}{|c|}
1 years: $1.38(0.84) ;$ \\
2 years: $1.05(0.99) ;$ \\
3 years: $1(1.02)$
\end{tabular} & \begin{tabular}{|l} 
1 years: $3.33(1.08) ;$ \\
2 years: $3.33(1.08) ;$ \\
3 years: $3.38(0.6)$ \\
\end{tabular} & \begin{tabular}{|l} 
years: $2.74(0.73) ;$ \\
2 years: $2.61(1.14) ;$ \\
3 years: $2.88(1.27)$
\end{tabular} \\
\hline Jung et al. [19] & 12 & 15 & 27 & 0 & 0 & 0 & 100 & 100 & \begin{tabular}{|c|}
5 years: \\
$1.5(0.8)$ mesial; \\
$1.7(0.7)$ distal \\
\end{tabular} & $\begin{array}{c}5 \text { years: } \\
1.4(0.5) \text { mesial; } \\
1.5(0.6) \text { distal } \\
\end{array}$ & \multicolumn{2}{|c|}{ is } & \multicolumn{2}{|c|}{ ניור } & \multicolumn{2}{|c|}{ J } & 5 years: $3.3(1.5)$ & 5 years: $3.7(1.2)$ \\
\hline Crespi et al. [20] & 197 & 78 & 275 & 2 & 0 & 2 & 98.9 & 100 & \begin{tabular}{|l|}
1 years: $0.77(0.39) ;$ \\
2 years: $0.82(0.52) ;$ \\
4 years: $0.79(0.38)$
\end{tabular} & $\begin{array}{l}1 \text { years: } 0.86(0.47) ; \\
2 \text { years: } 0.84(0.46) ; \\
4 \text { years: } 0.78(0.38) \\
\end{array}$ & \multicolumn{2}{|c|}{$\mathrm{N} / \mathrm{D}$} & \multicolumn{2}{|c|}{$\mathrm{N} / \mathrm{D}$} & 4 years: $0.78(0.23)$ & 4 years: $0.75(0.39)$ & \multicolumn{2}{|c|}{$\mathrm{N} / \mathrm{D}$} \\
\hline Crespi et al. [21] & 15 & 15 & 30 & 0 & 0 & 0 & 100 & 100 & \begin{tabular}{|l|}
1 years: $0.83(0.51) ;$ \\
2 years: $0.86(0.54)$
\end{tabular} & $\begin{array}{l}1 \text { years: } 0.80(0.47) ; \\
2 \text { years: } 0.82(0.52)\end{array}$ & $\begin{array}{r}1 \text { years: } 0.16(0.13) \text {; } \\
2 \text { years: } 0.2(0.13)\end{array}$ & $; \begin{array}{l}1 \text { years: } 0.21(0.13) ; \\
2 \text { years: } 0.25(0.18)\end{array}$ & \begin{tabular}{|l}
1 years: $1.8(0.64) ;$ \\
2 years: $1.99(0.57)$
\end{tabular} & \begin{tabular}{|l|l|} 
years: $1.85(0.68) ;$ \\
2 years: $2.05(0.66)$
\end{tabular} & \begin{tabular}{|l|} 
1 years: $0.69(0.3) ;$ \\
2 years: $0.72(0.36)$ \\
\end{tabular} & $\begin{array}{l}1 \text { years: } 0.68(0.34) ; \\
2 \text { years: } 0.77(0.33)\end{array}$ & $\begin{array}{l}1 \text { years: } 3.64(0.68) ; \\
2 \text { years: } 3.62(0.65)\end{array}$ & \begin{tabular}{|l}
1 years: $3.68(0.72) ;$ \\
2 years: $3.67(0.61)$
\end{tabular} \\
\hline Hita-Iglesias et al. [22] & 66 & 102 & 168 & 6 & 2 & 8 & 90.8 & 98.1 & \multirow{2}{*}{\multicolumn{2}{|c|}{\begin{tabular}{|l|}
$\mathrm{N} / \mathrm{D}$ \\
$\mathrm{N} / \mathrm{D}$ \\
\end{tabular}}} & \multicolumn{2}{|c|}{ N/D } & \multicolumn{2}{|c|}{$\mathrm{N} / \mathrm{D}$} & \multicolumn{2}{|c|}{$\mathrm{N} / \mathrm{D}$} & \multicolumn{2}{|c|}{$\begin{array}{l}\mathrm{N} / \mathrm{D} \\
\end{array}$} \\
\hline Blus et al. [23] & 83 & 85 & 168 & 2 & 1 & 3 & 97.6 & 98.8 & & & & //D & & & $\mathrm{N} / \mathrm{I}$ & & & \\
\hline Bell et al. [24] & 285 & 637 & 922 & 7 & 8 & 15 & 97.5 & 98.7 & \multicolumn{2}{|c|}{$\mathrm{N} / \mathrm{D}$} & \multicolumn{2}{|c|}{$\mathrm{N} / \mathrm{D}$} & \multicolumn{2}{|c|}{$\begin{array}{l}\text { N/D } \\
\text { N/D }\end{array}$} & \multicolumn{2}{|c|}{$\mathrm{N} / \mathrm{D}$} & \multicolumn{2}{|c|}{ N/D } \\
\hline Fugazzotto [25] & 64 & 64 & 128 & 1 & 1 & 2 & 98.1 & 98.2 & \multirow{2}{*}{\multicolumn{2}{|c|}{$\begin{array}{l}\frac{N / D}{N} \\
N / D\end{array}$}} & \multirow{2}{*}{\multicolumn{2}{|c|}{$\begin{array}{l}\frac{N / D}{N} \\
N / D\end{array}$}} & \multirow{2}{*}{\multicolumn{2}{|c|}{$\begin{array}{l}\frac{N / D}{N} \\
N / D\end{array}$}} & \multirow{2}{*}{\multicolumn{2}{|c|}{$\begin{array}{l}\text { N/D } \\
\text { N/D }\end{array}$}} & \multirow{2}{*}{\multicolumn{2}{|c|}{$\frac{\mathrm{N} / \mathrm{D}}{\mathrm{N} / \mathrm{D}}$}} \\
\hline Zuffetti et al. [26] & 193 & 334 & 527 & 3 & 7 & 10 & $98.4(0.9)$ & $97.9(0.8)$ & & & & & & & & & & \\
\hline
\end{tabular}

\begin{tabular}{lllllllllllll|l}
\hline Zuffetti et al. [26] & 193 & 334 & 527 & 3 & 7 & 10 & $98.4(0.9)$ & $97.9(0.8)$ & \\
\hline
\end{tabular}

$\mathrm{N} / \mathrm{D}$

$\mathrm{MBL}=$ marginal bone level; $\mathrm{MGL}=$ marginal gingival level changes; $\mathrm{PD}=$ probing depth; $\mathrm{WKG}=$ width of keratinized gingiva; $\mathrm{mBI}=$ modified bleeding index; $\mathrm{IS}=$ infected socket; $\mathrm{NIS}=$ non-infected socket; $\mathrm{N} / \mathrm{D}=$ no data; $\mathrm{SD}=$ standard deviation; $\mathrm{n}=$ numbers. 


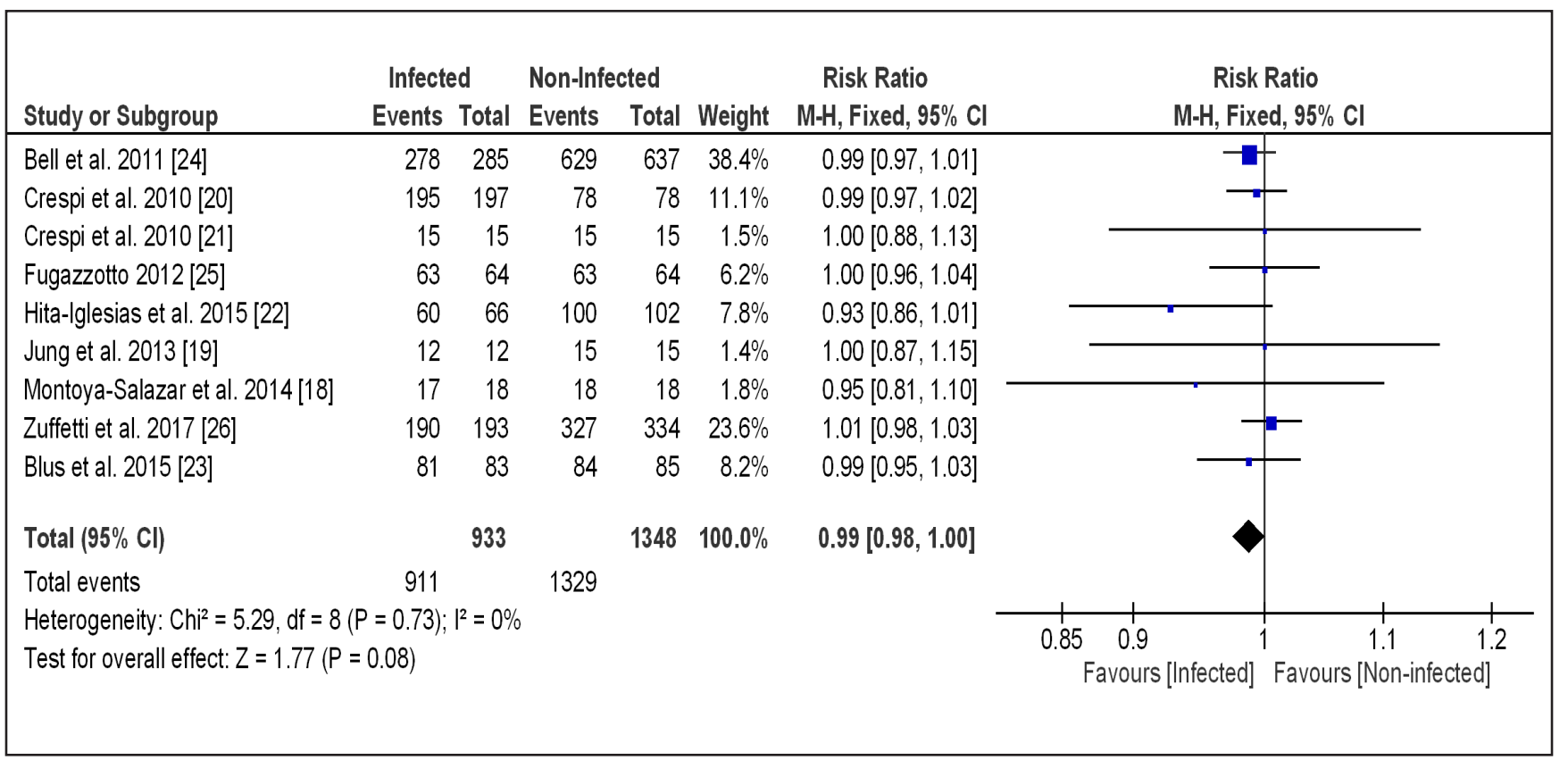

Figure 2. Implant survival rates of the included studies.

\begin{tabular}{|c|c|c|c|c|c|c|c|c|c|}
\hline \multirow{2}{*}{$\begin{array}{l}\text { Study or Subgroup } \\
\text { Bell et al } 2011 \text { [24] }\end{array}$} & $\begin{array}{l}\text { Infecte } \\
\text { Events }\end{array}$ & ed & $\begin{array}{l}\text { Non-infe } \\
\text { Events }\end{array}$ & $\begin{array}{l}\text { cted } \\
\text { Total }\end{array}$ & Weight & $\begin{array}{c}\text { Risk Ratio } \\
\text { M-H, Fixed, } 95 \% \mathrm{Cl}\end{array}$ & \multicolumn{3}{|c|}{$\begin{array}{c}\text { Risk Ratio } \\
\text { M-H, Fixed, } 95 \% \mathrm{Cl}\end{array}$} \\
\hline & 7 & 285 & 8 & 637 & $33.3 \%$ & $1.96[0.72,5.34]$ & & & \\
\hline Crespi et al. 2010 [20] & 2 & 197 & 0 & 78 & $4.8 \%$ & $1.99[0.10,41.09]$ & & & \\
\hline Crespi et al. 2010 [21] & 0 & 15 & 0 & 15 & & Not estimable & & & \\
\hline Fugazzotto 2012 [25] & 1 & 64 & 1 & 64 & $6.7 \%$ & $1.00[0.06,15.64]$ & & & \\
\hline Hita-Iglesias et al. 2015 [22] & 6 & 66 & 2 & 102 & $10.6 \%$ & $4.64[0.96,22.29]$ & & & \\
\hline Jung et al. 2013 [19] & 0 & 12 & 0 & 15 & & Not estimable & & & \\
\hline Montoya-Salazar et al. 2014 [18] & 1 & 18 & 0 & 18 & $3.4 \%$ & $3.00[0.13,69.09]$ & & & \\
\hline Zuffetti et al. 2017 [26] & 3 & 193 & 7 & 334 & $34.5 \%$ & $0.74[0.19,2.83]$ & & 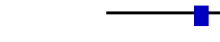 & r \\
\hline Blus et al. 2015 [23] & 2 & 83 & 1 & 85 & $6.7 \%$ & $2.05[0.19,22.16]$ & & & \\
\hline Total $(95 \% \mathrm{Cl})$ & & 933 & & 1348 & $100.0 \%$ & $1.80[0.98,3.31]$ & & & \\
\hline Total events & 22 & & 19 & & & & & & \\
\hline Heterogeneity: $\mathrm{Chi}^{2}=3.39, \mathrm{df}=6$ & $P=0.76) ;$ & $\left.\right|^{2}=0 \%$ & & & & & $\frac{1}{0.02}$ & 0.1 & 10 \\
\hline Test for overall effect: $Z=1.89$ (P & $0.06)$ & & & & & & & Favours [Infected] & ] Favours [Non-Infected] \\
\hline
\end{tabular}

Figure 3. Implant failure rates of the included studies.

\begin{tabular}{|c|c|c|c|c|c|c|c|c|c|}
\hline \multirow[b]{2}{*}{ Study or Subgroup } & \multicolumn{3}{|c|}{ Infected } & \multicolumn{3}{|c|}{ Non-Infected } & \multirow[b]{2}{*}{ Weight } & \multirow{2}{*}{$\begin{array}{l}\text { Mean Difference } \\
\text { IV, Random, } 95 \% \mathrm{Cl}\end{array}$} & \multirow{2}{*}{$\begin{array}{l}\text { Mean Difference } \\
\text { IV, Random, } 95 \% \mathrm{Cl}\end{array}$} \\
\hline & Mean & $S D$ & Total & Mean & SD & Total & & & \\
\hline Crespi et al. 2010 [20] & 0.77 & 0.39 & 197 & 0.86 & 0.47 & 78 & $62.4 \%$ & $-0.09[-0.21,0.03]$ & -1 \\
\hline Crespi et al. 2010 [21] & 0.83 & 0.51 & 15 & 0.8 & 0.47 & 15 & $7.0 \%$ & $0.03[-0.32,0.38]$ & \\
\hline Montoya-Salazar et al. 2014 [18] & 0.73 & 0.22 & 18 & 0.73 & 0.29 & 18 & $30.6 \%$ & $0.00[-0.17,0.17]$ & \\
\hline Total $(95 \% \mathrm{Cl})$ & & & 230 & & & 111 & $100.0 \%$ & $-0.05[-0.15,0.04]$ & \\
\hline \multicolumn{9}{|c|}{$\begin{array}{l}\text { Heterogeneity: } \text { Tau }^{2}=0.00 ; \mathrm{Chi}^{2}=0.98, \mathrm{df}=2(P=0.61) ; I^{2}=0 \% \\
\text { Test for overall effect: } Z=1.14(P=0.25)\end{array}$} & $\begin{array}{ccccc}-0.5 & -0.25 & 0 & 0.25 & 0.5 \\
\text { Favours [Infected] } & \text { Favours [Non-Infected] }\end{array}$ \\
\hline
\end{tabular}

Figure 4A. Marginal bone level changes at the follow-up period of 1 year. 


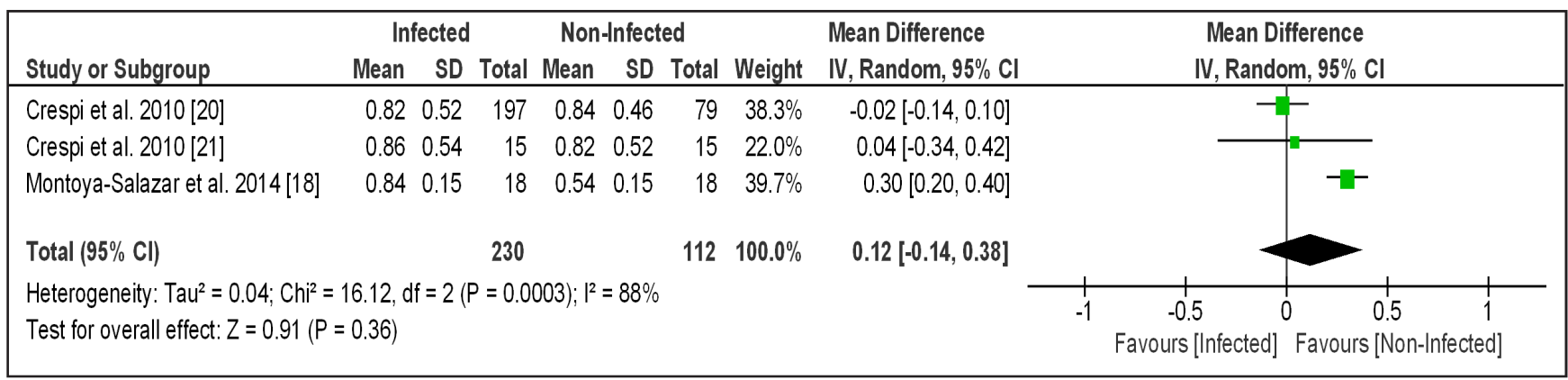

Figure 4B. Marginal bone level changes at the follow-up period of 2 years.

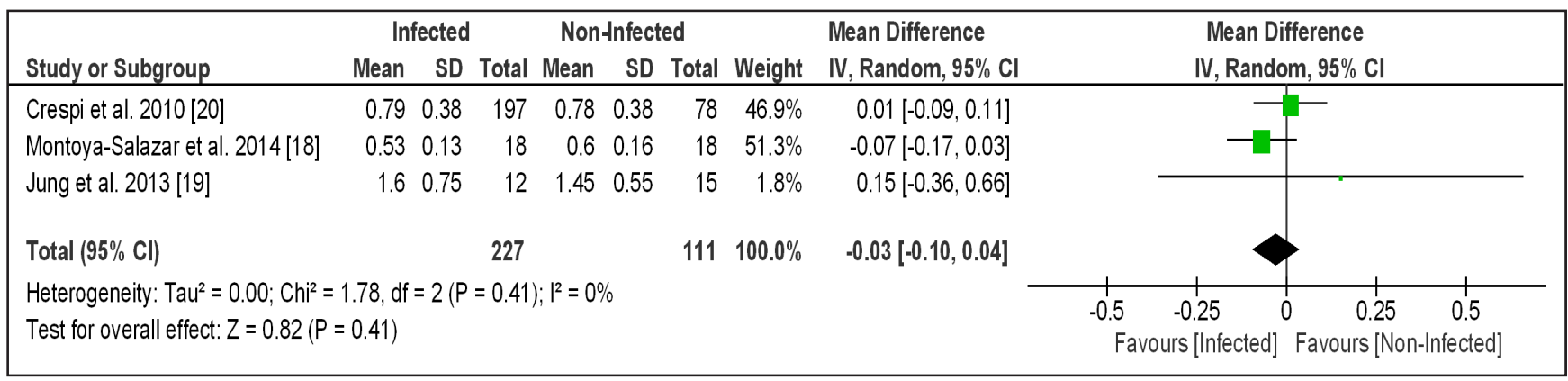

Figure 4C. Marginal bone level changes at the follow-up period of $>3$ years.

\begin{tabular}{|c|c|c|c|c|c|c|c|c|c|}
\hline \multirow[b]{2}{*}{ Study or Subgroup } & \multicolumn{3}{|c|}{ Infected } & \multicolumn{3}{|c|}{ Non-Infected } & \multicolumn{2}{|r|}{ Mean Difference } & \multirow{2}{*}{$\begin{array}{l}\text { Mean Difference } \\
\text { IV, Fixed, } 95 \% \mathrm{Cl}\end{array}$} \\
\hline & Mean & SD & Total & Mean & $S D$ & Total & Weight & IV, Fixed, $95 \% \mathrm{Cl}$ & \\
\hline Crespi et al. 2010 [21] & 0.16 & 0.13 & 15 & 0.21 & 0.13 & 15 & $93.8 \%$ & $-0.05[-0.14,0.04]$ & \\
\hline Montoya-Salazar et al. 2014 [18] & 0.88 & 0.75 & 18 & 1.13 & 0.23 & 18 & $6.2 \%$ & $-0.25[-0.61,0.11]$ & \\
\hline Total $(95 \% \mathrm{Cl})$ & & & 33 & & & 33 & $100.0 \%$ & $-0.06[-0.15,0.03]$ & \\
\hline \multicolumn{9}{|c|}{$\begin{array}{l}\text { Heterogeneity: } \text { Chi }^{2}=1.10, d f=1(P=0.29) ; l^{2}=9 \% \\
\text { Test for overall effect: } Z=1.36(P=0.17)\end{array}$} & $\begin{array}{ccccc}-0.5 & -0.25 & 0 & 0.25 & 0.5 \\
\text { Favours [Infected] } & \text { Favours [Non-Infected] }\end{array}$ \\
\hline
\end{tabular}

Figure 5A. Marginal gingival level changes at the follow-up period of 1 year.

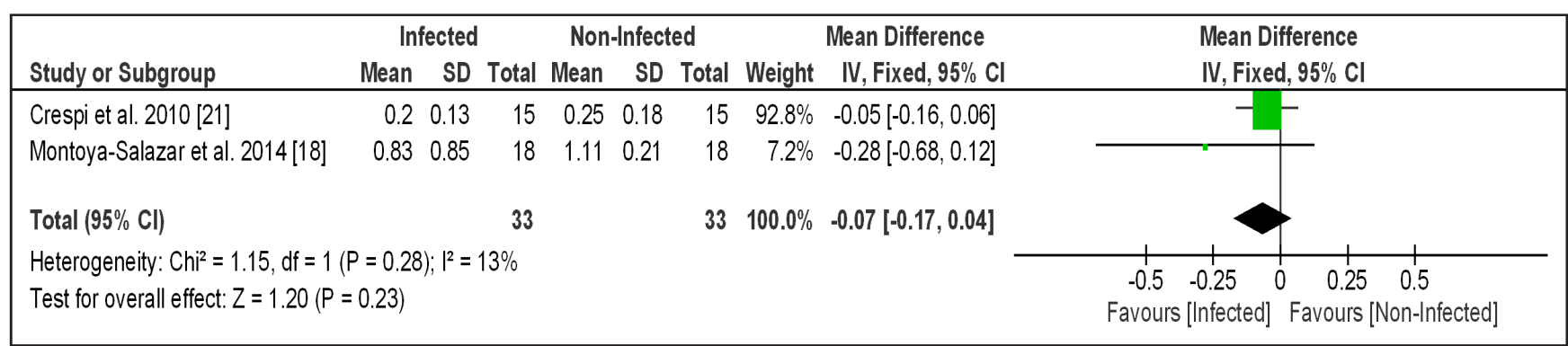

Figure 5B. Marginal gingival level changes at the follow-up period of 2 years.

\begin{tabular}{|c|c|c|c|c|c|c|c|c|c|}
\hline \multirow[b]{2}{*}{ Study or Subgroup } & \multicolumn{3}{|c|}{ Infected } & \multicolumn{3}{|c|}{ Non-Infected } & \multicolumn{2}{|c|}{ Mean Difference } & \multirow{2}{*}{$\begin{array}{l}\text { Mean Difference } \\
\text { IV, Fixed, } 95 \% \mathrm{Cl}\end{array}$} \\
\hline & Mean & SD & Total & Mean & SD & Total & Weight & IV, Fixed, $95 \% \mathrm{Cl}$ & \\
\hline Crespi et al. 2010 [21] & 1.8 & 0.64 & 15 & 1.85 & 0.68 & 15 & $20.6 \%$ & $-0.05[-0.52,0.42]$ & \\
\hline Montoya-Salazar et al. 2014 [18] & 2.53 & 0.44 & 18 & 2.44 & 0.28 & 18 & $79.4 \%$ & $0.09[-0.15,0.33]$ & \\
\hline Total $(95 \% \mathrm{Cl})$ & & & 33 & & & 33 & $100.0 \%$ & $0.06[-0.15,0.28]$ & \\
\hline \multicolumn{9}{|c|}{$\begin{array}{l}\text { Heterogeneity: } \text { Chi }^{2}=0.27, d f=1(P=0.60) ; I^{2}=0 \% \\
\text { Test for overall effect: } Z=0.56(P=0.58)\end{array}$} & $\begin{array}{ccccc}-0.5 & -0.25 & 0 & 0.25 & 0.5 \\
\text { Favours [Infected] } & \text { Favours [Non-Infected] }\end{array}$ \\
\hline
\end{tabular}

Figure 6A. Probing depth changes of the included studies at the follow-up period of 1 year. 


\begin{tabular}{|c|c|c|c|c|c|c|c|c|c|}
\hline \multirow[b]{2}{*}{ Study or Subgroup } & \multicolumn{3}{|c|}{ Infected } & \multicolumn{3}{|c|}{ Non-Infected } & \multicolumn{2}{|r|}{ Mean Difference } & \multirow{2}{*}{$\begin{array}{l}\text { Mean Difference } \\
\mathrm{IV} \text {, Fixed, } 95 \% \mathrm{Cl}\end{array}$} \\
\hline & Mean & SD & Total & Mean & SD & Total & Weight & IV, Fixed, $95 \% \mathrm{Cl}$ & \\
\hline Crespi et al. 2010 [21] & 1.99 & 0.57 & 15 & 2.05 & 0.66 & 15 & $46.0 \%$ & $-0.06[-0.50,0.38]$ & \\
\hline Montoya-Salazar et al. 2014 [18] & 2.76 & 0.8 & 18 & 2.6 & 0.37 & 18 & $54.0 \%$ & $0.16[-0.25,0.57]$ & \\
\hline Total $(95 \% \mathrm{Cl})$ & & & 33 & & & 33 & $100.0 \%$ & $0.06[-0.24,0.36]$ & \\
\hline \multicolumn{4}{|c|}{$\begin{array}{l}\text { Heterogeneity: } \mathrm{Chi}^{2}=0.52, \mathrm{df}=1(P=0.47) ; \mathrm{I}^{2}=0 \% \\
\text { Test for overall effect: } Z=0.39(P=0.70)\end{array}$} & & & & & & $\begin{array}{ccccc}-0.5 & -0.25 & 0 & 0.25 & 0.5 \\
\text { Favours [Infected] } & \text { Favours [Non-Infected] }\end{array}$ \\
\hline
\end{tabular}

Figure 6B. Probing depth changes of the included studies at the follow-up-period of 2 years.

No significant difference was found between the groups at follow-up, at year 1 the MD was 0.06 (95\% $\mathrm{CI}=-0.15$ to $0.28 ; \mathrm{P}=0.58)$ and at year 2 the $\mathrm{MD}$ was $0.06(95 \% \mathrm{CI}=-0.24$ to $0.36 ; \mathrm{P}=0.7)$.

\section{Modified bleeding index changes}

Three of the included clinical trials analyzed the $\mathrm{mBI}$ (Figure 7A - C) $[\underline{18}, \underline{20}, \underline{21}]$. One of the studies reported three follow-up periods of 1 year, 2 years and 3 years [20]. Another one reported two followup periods of 1 and 2 years [21] and the remaining study included only a 4 year follow-up [20]. Both of the 1 year and 2 year analysis included 33 implants in both infected and non-infected groups. The 3 year or more follow-up included 215 implants in the infected sockets and 96 implants in the non-infected sockets. No statistical significant difference was seen between the groups at different follow-up times, at year 1 the MD was $-0.07(95 \% \mathrm{CI}=-0.28$ to $0.14 ; \mathrm{P}=0.5)$, at year 2 the MD was $-0.07(95 \% \mathrm{CI}=-0.3$ to 0.15 ;
$\mathrm{P}=0.52$ ) and at year 3 or more the MD was $-0.00162196(95 \% \mathrm{CI}=-0.09$ to $0.09 ; \mathrm{P}=0.97)$.

\section{Width of keratinized gingival changes}

Three clinical trials $[18,20,21]$ analyzed changes of WKG. One of the studies reported three follow-up periods of 1 year, 2 years and 3 years [18]. Another one reported two follow-up periods of 1 year and 2 years [21] and the remaining study included one follow-up period of 5 year [19] (Figure 8A - C). Both of the 1 year and 2 year analysis included 33 implants in both infected and non-infected groups. The 3 year or more follow-up period included 30 implants in the infected group and 33 implants in the non-infected group. There was a slight, but significant decrease of WKG which favoured the non-infected group (1 year, $\mathrm{MD}=0.22 ; 95 \% \mathrm{CI}=-0.17$ to $0.6 ; \mathrm{P}=0.27$; at year $2, \mathrm{MD}=0.16 ; 95 \% \mathrm{CI}=-0.22$ to $0.55 ; \mathrm{P}=0.4$; and at 3 years or more, $\mathrm{MD}=0.25 ; 95 \% \mathrm{CI}=-0.3$ to 0.8 ; $\mathrm{P}=0.38)$.

\begin{tabular}{|c|c|c|c|c|c|c|c|c|c|}
\hline \multirow[b]{2}{*}{ Study or Subgroup } & \multicolumn{3}{|c|}{ Infected } & \multicolumn{3}{|c|}{ Non-Infected } & \multicolumn{2}{|r|}{ Mean Difference } & \multirow{2}{*}{$\begin{array}{l}\text { Mean Difference } \\
\text { IV, Fixed, } 95 \% \mathrm{Cl}\end{array}$} \\
\hline & Mean & SD & Total & Mean & SD & Total & Weight & IV, Fixed, $95 \% \mathrm{Cl}$ & \\
\hline Crespi et al. 2010 [21] & 0.69 & 0.3 & 15 & 0.68 & 0.34 & 15 & $83.7 \%$ & $0.01[-0.22,0.24]$ & 一 \\
\hline Montoya-Salazar et al. 2014 [18] & 0.88 & 0.75 & 18 & 1.38 & 0.84 & 18 & $16.3 \%$ & $-0.50[-1.02,0.02]$ & \\
\hline Total $(95 \% \mathrm{Cl})$ & & & 33 & & & 33 & $100.0 \%$ & $-0.07[-0.28,0.14]$ & \\
\hline \multicolumn{4}{|c|}{$\begin{array}{l}\text { Heterogeneity: } \text { Chi' }^{2}=3.09, d f=1(P=0.08) ;\left.\right|^{2}=68 \% \\
\text { Test for overall effect: } Z=0.68(P=0.50)\end{array}$} & & & & & & $\begin{array}{ccccc}-1 & -0.5 & 0 & 0.5 & 1 \\
\text { Favours [Infected] } & \text { Favours [Non-Infected] }\end{array}$ \\
\hline
\end{tabular}

Figure 7A. Modified bleeding index changes of the included studies at the follow-up period of 1 year.

\begin{tabular}{|c|c|c|c|c|c|c|c|c|c|}
\hline \multirow[b]{2}{*}{ Study or Subgroup } & \multicolumn{3}{|c|}{ Infected } & \multicolumn{3}{|c|}{ Non-Infected } & \multicolumn{2}{|c|}{ Mean Difference } & \multirow{2}{*}{$\begin{array}{l}\text { Mean Difference } \\
\text { IV, Fixed, } 95 \% \mathrm{Cl}\end{array}$} \\
\hline & Mean & SD & Total & Mean & SD & Total & Weight & IV, Fixed, $95 \% \mathrm{Cl}$ & \\
\hline Crespi et al. 2010 [21] & 0.72 & 0.36 & 15 & 0.77 & 0.33 & 15 & $85.6 \%$ & $-0.05[-0.30,0.20]$ & - \\
\hline Montoya-Salazar et al. 2014 [18] & 0.83 & 0.85 & 18 & 1.05 & 0.99 & 18 & $14.4 \%$ & $-0.22[-0.82,0.38]$ & \\
\hline Total $(95 \% \mathrm{Cl})$ & & & 33 & & & 33 & $100.0 \%$ & $-0.07[-0.30,0.15]$ & \\
\hline \multicolumn{4}{|c|}{ Heterogeneity: $\mathrm{Chi}^{2}=0.26, d f=1(P=0.61) ;\left.\right|^{2}=0 \%$} & \multicolumn{5}{|c|}{ Test for overall effect: $Z=0.64(P=0.52)$} & $\begin{array}{ccccc}-1 & -0.5 & 0 & 0.5 & 1 \\
\text { Favours } & \text { [nfected] } & \text { Favours [Non-Infected] }\end{array}$ \\
\hline
\end{tabular}

Figure 7B. Modified bleeding index changes of the included studies at the follow-up period of 2 years. 


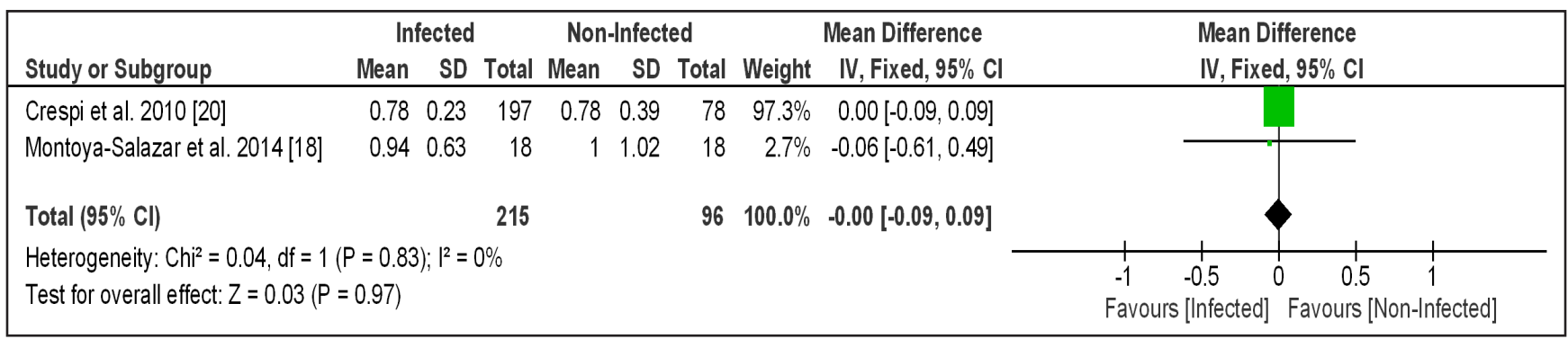

Figure 7C. Modified bleeding index changes of the included studies at the follow-up period of $>3$ years.

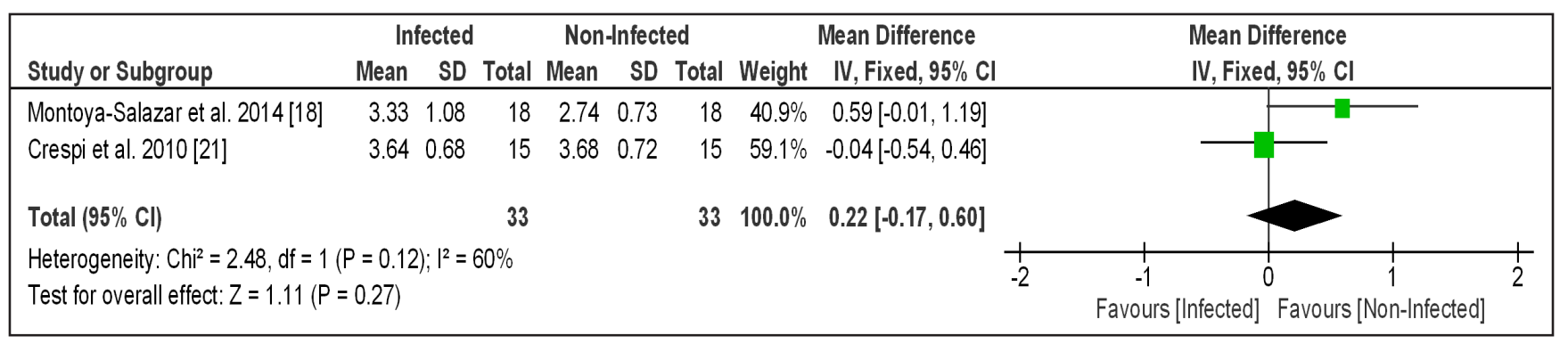

Figure 8A. Width of keratinized gingiva of the included studies at the follow-up period of 1 year.

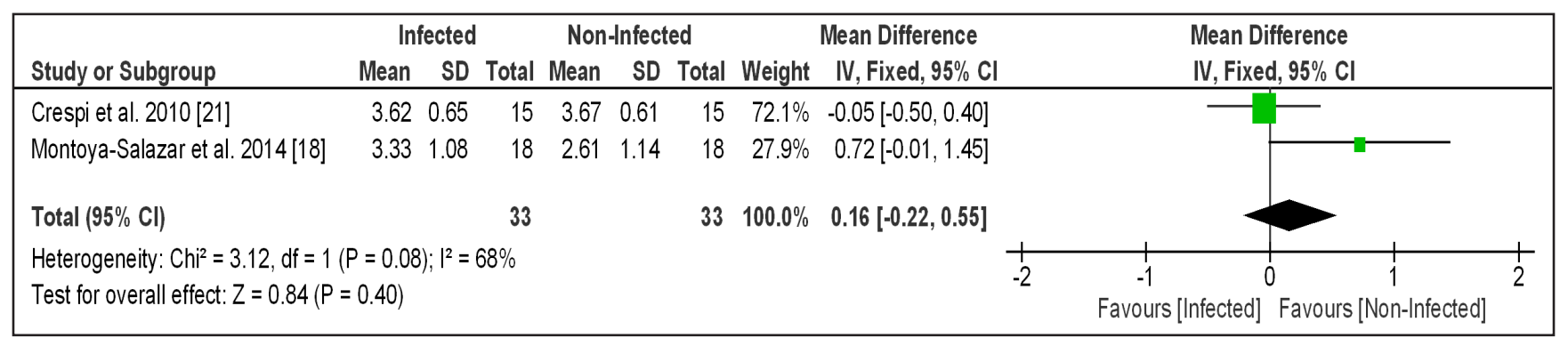

Figure 8B. Width of keratinized gingiva of the included studies at the follow-up period of 2 years.

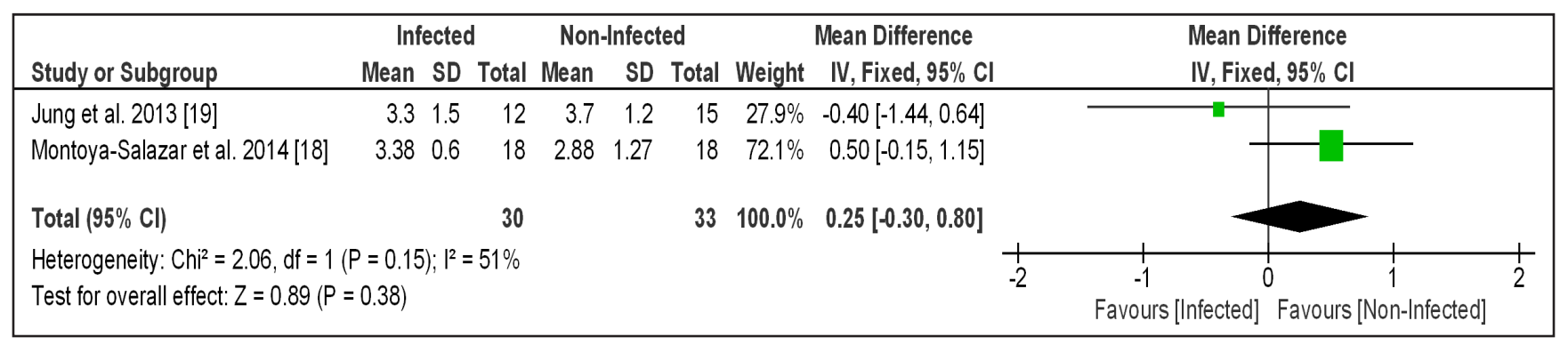

Figure 8C. Width of keratinized gingiva of the included studies at the follow-up period of $>3$ years.

\section{Publication bias}

No obvious visual publication bias was observed in the funnel plot analysing the implant survival rate (primary outcome variable) seen in Figure 9.

\section{DISCUSSION \\ Principal findings}

The results of this meta-analysis suggest that there is no statistical significant difference in implant survival rates $(\mathrm{RR}=0.99 ; 95 \% \mathrm{CI}=0.98$ to $1 ; \mathrm{P}=0.08)$ between immediate implant placement in infected sockets and non-infected sockets. Furthermore, all of the secondary outcome variables (MBL, MGL, mBI and PD) showed equal favourable results except for WKG that showed a slight but significant decrease in the infected group.

Implant survival depends on the MBL changes and is one of the factors to determine the success of the implant survival $[\underline{30}, \underline{31}]$. In this present review no significant difference was found between the two groups ( 1 year, $\mathrm{MD}=-0.05 ; 95 \% \mathrm{CI}=-0.15$ to 0.04 ; 


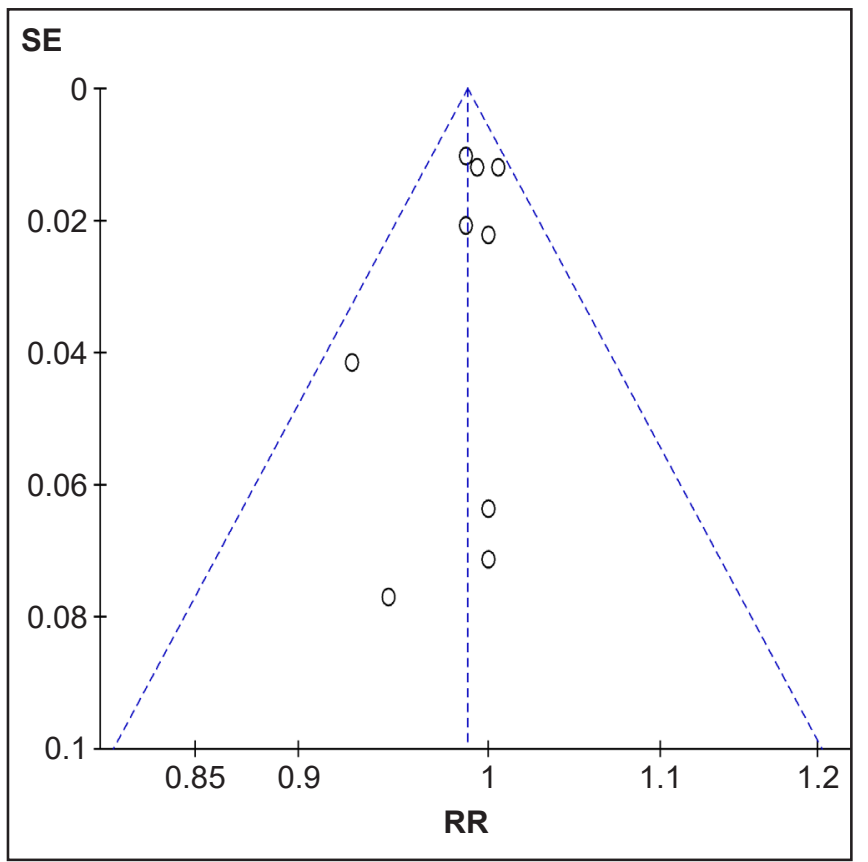

Figure 9. Funnel plot demonstrating publication bias. $\mathrm{SE}=$ standard error; $\mathrm{RR}=$ risk ratio.

$\mathrm{P}=0.25$; year $2, \mathrm{MD}=0.12 ; 95 \% \mathrm{CI}=-0.14$ to $0.38 ; \mathrm{P}=0.36 ;>3$ years, $\mathrm{MD}=-0.03 ; 95 \% \mathrm{CI}=$ -0.1 to $0.04 ; \mathrm{P}=0.41)$. Albrektsson and Isidor [32] suggested that implant success is valid if less than $1.5 \mathrm{~mm}$ of bone loss is seen during the first year after functional loading and thereafter a loss of $<0.2 \mathrm{~mm}$ annually. Thus, meaning that marginal bone loss is inevitable. Early MBL changes are a type of adaptive non-infective process that is influenced by surgical factors (surgical trauma, bone overheating, excessive implant tightening and crestal width) and prosthetic trauma (occlusial overload, type of implant design, microgap, abutment height and foreign body reaction to cement residue) $[\underline{30}, \underline{34}, \underline{35}]$. A study done by Galindo-Moreno et al. [34] found that early high MBL changes of $0.44 \mathrm{~mm}$ at six months (after loading) were strongly associated with a subsequent increase of MBL changes of $>2 \mathrm{~mm}$ at 18 months. Hence, this six month period may be used as an indicator for long term bone loss prognosis.

Most of the included studies used guided bone regeneration (GBR) as a type of treatment method. The buccal bone plate can undergo more than $50 \%$ of horizontal reduction following the placement of immediate implants; this can lead to gingival recession, impairing the aesthetics. Additionally, a mean of $1 \mathrm{~mm}$ vertical bone loss can be seen in the presence of a thin buccal bone $[\underline{35}, \underline{36}]$. In this study no significant changes of MGL were seen between the groups (year $1, \mathrm{MD}=-0.06 ; 95 \% \mathrm{CI}=-0.15$ to 0.03 ; $\mathrm{P}=0.17$; year $2, \mathrm{MD}=-0.07 ; 95 \% \mathrm{CI}=-0.17$ to 0.04 ; $\mathrm{P}=0.23)$.
The structural characteristics of the gingiva have been considered important for the integrity of the periodontium. A movable gingival margin facilitates the introduction of biofilm into the gingival crevice, resulting in sub-gingival plaque that triggers the activation of lymphocytes and neutrophils. This biofilm penetration induces a chronic inflammatory response [37]. However, as there are anatomical and structural differences between natural dentition and implants, the same consensus might not be applicable. The significance of the keratinized mucosa on periimplant health has been widely discussed [38]. A study done by Pranskunas et al. [39] concluded that implants with narrow WKG $(<2 \mathrm{~mm})$ had significantly more plaque, signs of inflammation, decreased stability of peri-implant site and increased mucosal recession than those with wider WKG $(>2$ $\mathrm{mm})$. These finding are supported by other studies $[\underline{37}, \underline{38}, \underline{40}]$. However, when adequate plaque control is followed, data suggests no correlation between WKG and peri-implant conditions [37-40]. On the other hand, Monje and Blasi [37] found a correlation between narrow keratinized mucosa and a decrease in vestibular depth - which may impair patients' ability to implement correct oral hygiene measures. Additionally, WKG of $<2 \mathrm{~mm}$ is associated with increased brushing discomfort and as well as inadequate aesthetical outcome [39-41]. The present review and meta-analysis showed that a slight but significant amount of WKG (1 year, MD $=0.22 ; 95 \%$ $\mathrm{CI}=-0.17$ to $0.6 ; \mathrm{P}=0.27$; at year $2, \mathrm{MD}=0.16 ; 95 \%$ $\mathrm{CI}=-0.22$ to $0.55 ; \mathrm{P}=0.4$; and at 3 years or more, $\mathrm{MD}=0.25 ; 95 \% \mathrm{CI}=-0.3$ to $0.8 ; \mathrm{P}=0.38)$ is lost during the immediate implant placement in infected sites. This suggests that WKG should be of concern in clinical situations were optimum plaque control is not feasible or when there is a high aesthetic demand.

Additionally, PD and $\mathrm{mBI}$ clinical parameters were collected and compared to determine if there were any soft tissue changes indicating inflammation. The two groups showed no significant statistical differences in both of the analyses; PD (year $1, \mathrm{MD}=0.06 ; 95 \% \mathrm{CI}$ $=-0.15$ to $0.28 ; \mathrm{P}=0.58$; and year $2, \mathrm{MD}=0.06 ; 95 \%$ $\mathrm{CI}=-0.24$ to $0.36 ; \mathrm{P}=0.7$ ) and $\mathrm{mBI}$ (year $1, \mathrm{MD}=$ $-0.07 ; 95 \% \mathrm{CI}=-0.28$ to $0.14 ; \mathrm{P}=0.5$; year $2, \mathrm{MD}=$ $-0.07 ; 95 \% \mathrm{CI}=-0.3$ to $0.15 ; \mathrm{P}=0.52$ and year 3 or more, $\mathrm{MD}=-0.00162196 ; 95 \% \mathrm{CI}=-0.09$ to 0.09 ; $\mathrm{P}=0.97)$.

Immediate loading was used in some of the included studies. In the review conducted by Pigozzo et al. [42] showed that both immediate and early loading protocols in single implant crowns had high success rate. Another study done by Gallucci et al. [43] showed a survival rate of $98.4 \%$ and a success rate of 
$87 \%$ to $100 \%$ in immediate implant placement with immediate loading.

All of the included studies reported about removing of the granulation tissue before the implant placement. In general, most studies recommend curettage of the implant site before placement or suggest antibiotics to aid the success rate of immediate implant placement [44]. During the primary stability, the outer implant threads are in close proximity with the surrounding bone, providing mechanical interlocking between the bone and implant $[\underline{45}, \underline{46}]$. However, the inner surfaces of the threads are unable to have an implantto-bone contact and the void formed will be occupied with blood, subsequently forming into a blood clot characterized by a fibrin coagulum with thrombocytes, neutrophils, erythrocytes and macrophages/ monocytes [45]. The fibrin coagulum network will progressively form into granulation tissue when penetration of vascular units and fibroblast-like cells is initiated. This initial wound healing response will start the bone apposition between the implant and the surrounding bone, indicating the build-up of the secondary stability $[\underline{45}, \underline{46}]$. On the contrary, when in presence of infection, sites showing pathology may increase the risk of microbial interference with the initial wound healing $[47,48]$. Even after vigorous curettage and irrigation of the infected socket, some microbial pathogenic species are able to survive in a vegetative state at the site and once the implant is placed they might reactivate and colonize the implant surface initiating retrograde peri-implantitis and bone loss [48-50]. However, recent evidence suggests that granulation tissue collected from infected sites behave similarly to granulation tissue of healing wounds. The findings imply that the cell cultures taken from these granulation tissues contain pluripotent stem cells that might aid tissue healing if the infection is controlled [48]. The study done by Crespi et al. [48] compared two infected socket groups: for one group debridement was performed, and for the second group the granulation tissue was left. The results showed equal favourable outcomes for both of the groups after a period of one year. Although the study has showed favourable results, more long-term randomized clinical trials evaluating clinical and histological results are needed as the data is very limited.

All of the included clinical trials used either preoperative, postoperative or both antibiotic prophylaxes as a treatment protocol. The usage of antibiotics as a preventive measurement in healthy patients for suppressing the residual infection left during debridement, postoperative infections and oral implant failures is still disputed [51]. Questions about the type of antibiotics, the dosage and regimen to follow still remain. Romandini et al. [52] found in their review that all introduced protocols reduced early implant failures; however, postoperative prescription due to its prolonged course was associated with higher adverse events (resistance). Nonetheless, further research is needed for a definite protocol.

\section{Previous systematic reviews}

The findings of this present meta-analysis are similar to the two previous meta-analyses done on this subject $[53,54]$. Both of those studies indicated that the placement of immediate implants into infected sockets does not significantly affect the rate of implant survival. The quantitative analyses performed by Lee et al. [53] included only three of the studies presented in this review $[18,19,21]$. Clinical parameters such as MBL, MGL, PD, $\mathrm{mBI}$ and $\mathrm{WKG}$ were included in the meta-analysis. However, separate analyses for different follow-up periods to decrease the heterogeneity were not made; only the latest followup periods were compared. The meta-analysis done by Chen et al. [54] included all of the studies presented in this review but exclusion was made on all implants that were not placed in the aesthetic zone. Clinical parameters such as MBL and MGL were included; however, meta-analysis was not conducted on PD, $\mathrm{mBI}$ and $\mathrm{WKG}$.

\section{Limitations and future scientific recommendations}

It is important to highlight that no randomized clinical trials were found concerning this subject. It may not be feasible to compare these protocols for the reason that it can be quite challenging to apply similar selection criteria as there are many cases that present with extraction site risk factors such as presence of thin or absent buccal bone, making them less preferable for one protocol. In addition, only English studies were included, a factor that can cause bias with paper selection due to exclusion of any possible primary studies in other languages.

Numerous scientific studies made on osseointegrated implants use the terms "implant survival" and "implant success" synonymously which can generate confusion. It is of importance to differentiate these two terms in order to facilitate the same quality and outcome data within all studies. "Implant survival" is defined as implant and fixed prosthesis present in the mouth regardless of biological and technical complications, while "implant success" involves both clinical (PD, mBI, and modified plaque index) and radiological aspects [55-57]. 
The survival rates of modern implantology are considered high and predictable. Hence, additional criteria such as the aesthetics of the peri-implant soft tissues have become one of the important factors to evaluate the implant success criteria $[\underline{2}, \underline{7}, \underline{8}]$. Bone characteristics and soft tissue dimensions are critical factors in achieving a satisfactory aesthetic outcome $[1,12]$. Therefore, a classification of the fresh-sockets including both soft and hard tissues is necessary [58]. The authors Juodzbalys et al. [두] for example, have proposed a classification system and treatment recommendations incorporating both soft and hard tissues for immediate implant placement. The classifications assessing the fresh-socket morphology are helpful tools for the clinician to plan and judge future clinical situations. Only few of the included studies documented clinical and radiographic parameters such as presence of keratinized mucosa, PD, attachment level, plaque index, bleeding index, MBL, MGL and the peri-implant maintenance therapy. Furthermore, classification of the socket pathology origin was vague and varied among the studies. For future investigation, clinical trials should be conducted with proper documentation of the fresh-socket site morphology and origin of pathology as well as treatment and measurement procedures together with appropriately set inclusion criteria.

\section{CONCLUSIONS}

The conducted meta-analysis suggests that there is no statistical significant difference in survival rates between infected sockets and non-infected sockets. All of the secondary outcome variables showed equal favourable results, except for width of keratinized gingiva, which slightly but significantly favoured the non-infected group. However, randomized controlled clinical trials with large samples should be made in order to draw a definite conclusion about the efficacy and safety of the treatment.

\section{ACKNOWLEDGEMENTS AND DISCLOSURE STATEMENTS}

The authors confirm no conflicts of interest related to this systematic review.

\section{REFERENCES}

1. Blanco J, Carral C, Argibay O, Liñares A. Implant placement in fresh extraction sockets. Periodontol 2000. 2019 Feb;79(1):151-167. [Medline: 30892772] [doi: 10.1111/prd.12253]

2. Ebenezer V, Balakrishnan K, Asir RV, Sragunar B. Immediate placement of endosseous implants into the extraction sockets. J Pharm Bioallied Sci. 2015 Apr;7(Suppl 1):S234-7. [Medline: 26015721] [PMC free article: 4439681] [doi: 10.4103/0975-7406.155926]

3. Bassir SH, El Kholy K, Chen CY, Lee KH, Intini G. Outcome of early dental implant placement versus other dental implant placement protocols: A systematic review and meta-analysis. J Periodontol. 2019 May;90(5):493-506. [Medline: 30395355] [PMC free article: 6500770] [doi: 10.1002/JPER.18-0338]

4. Jyothi SG, Triveni MG, Mehta DS, Nandakumar K. Evaluation of single-tooth replacement by an immediate implant covered with connective tissue graft as a biologic barrier. J Indian Soc Periodontol. 2013 May;17(3):354-60. [Medline: 24049337] [PMC free article: 3768187] [doi: 10.4103/0972-124X.115666]

5. Mello CC, Lemos CAA, Verri FR, Dos Santos DM, Goiato MC, Pellizzer EP. Immediate implant placement into fresh extraction sockets versus delayed implants into healed sockets: A systematic review and meta-analysis. Int J Oral Maxillofac Surg. 2017 Sep;46(9):1162-1177. [Medline: 28478869] [doi: 10.1016/j.ijom.2017.03.016]

6. Canellas JVDS, Medeiros PJD, Figueredo CMDS, Fischer RG, Ritto FG. Which is the best choice after tooth extraction, immediate implant placement or delayed placement with alveolar ridge preservation? A systematic review and metaanalysis. J Craniomaxillofac Surg. 2019 Nov;47(11):1793-1802. [Medline: 31522823] [doi: 10.1016/j.jcms.2019.08.004]

7. Swathi KV. Immediate implant placement-A review. J Pharm Sci Res. 2016;8(11): 1315-7. [URL: https://www.jpsr.pharmainfo.in]

8. Waasdorp JA. Er,Cr:YSGG Laser Debridement of an Infected Socket for Immediate Implant Placement: A Case Report. Clin Adv Periodontics. 2018 Apr; 8(3): 115-9. [doi: 10.1002/cap.10023]

9. Ortega-Martínez J, Pérez-Pascual T, Mareque-Bueno S, Hernández-Alfaro F, Ferrés-Padró E. Immediate implants following tooth extraction. A systematic review. Med Oral Patol Oral Cir Bucal. 2012 Mar 1;17(2):e251-61. [Medline: 22143704] [PMC free article: 3448306] [doi: 10.4317/medoral.17469]

10. Tonetti MS, Cortellini P, Graziani F, Cairo F, Lang NP, Abundo R, Conforti GP, Marquardt S, Rasperini G, Silvestri M, Wallkamm B, Wetzel A. Immediate versus delayed implant placement after anterior single tooth extraction: the timing randomized controlled clinical trial. J Clin Periodontol. 2017 Feb;44(2):215-224. [Medline: 27978602] [doi: $10.1111 /$ jcpe.12666] 
11. Liu R, Yang Z, Tan J, Chen L, Liu H, Yang J. Immediate implant placement for a single anterior maxillary tooth with a facial bone wall defect: A prospective clinical study with a one-year follow-up period. Clin Implant Dent Relat Res. 2019 Dec;21(6):1164-1174. [Medline: 31709704] [doi: 10.1111/cid.12854]

12. Clementini M, Agostinelli A, Castelluzzo W, Cugnata F, Vignoletti F, De Sanctis M. The effect of immediate implant placement on alveolar ridge preservation compared to spontaneous healing after tooth extraction: Radiographic results of a randomized controlled clinical trial. J Clin Periodontol. 2019 Jul;46(7):776-786. [Medline: 31050359] [doi: $10.1111 /$ jepe.13125]

13. Furtado Araújo MV, Sommerville D, Dhingra A, Schincaglia GP. Immediate Placement and Restoration of an Implant in an Infected Socket in the Esthetic Zone. Clin Adv Periodontics. 2015 May;5(2): 83-9. [doi: 10.1902/cap.2013.130010]

14. Kim JJ, Song HY, Ben Amara H, Kyung-Rim K, Koo KT. Hyaluronic Acid Improves Bone Formation in Extraction Sockets With Chronic Pathology: A Pilot Study in Dogs. J Periodontol. 2016 Jul;87(7):790-5. [Medline: 26991484] [doi: 10.1902/jop.2016.150707]

15. Moher D, Liberati A, Tetzlaff J, Altman DG; PRISMA Group. Preferred reporting items for systematic reviews and meta-analyses: the PRISMA statement. Int J Surg. 2010;8(5):336-41. doi: 10.1016/j.ijsu.2010.02.007. Epub 2010 Feb 18. Erratum in: Int J Surg. 2010;8(8):658. [Medline: 20171303] [doi: 10.1016/j.ijsu.2010.02.007]

16. Higgins JPT, Altman DG, Sterne JAC. Chapter 8: assessing risk of bias in included studies. In: Higgins JPT, Green S, editors. Cochrane handbook for systematic reviews of interventions version 5.1.0 (updated March 2011). The Cochrane Collaboration. 2011. [URL: http://handbook.cochrane.org/]

17. Moola S, Munn Z, Tufanaru C, Aromataris E, Sears K, Sfetcu R, Currie M, Qureshi R, Mattis P, Lisy K, Mu P-F. Chapter 7: Systematic reviews of etiology and risk. In: Aromataris E, Munn Z, editors. Joanna Briggs Institute Reviewer's Manual. The Joanna Briggs Institute. 2017. [URL: https://reviewersmanual.joannabriggs.org/]

18. Montoya-Salazar V, Castillo-Oyagüe R, Torres-Sánchez C, Lynch CD, Gutiérrez-Pérez JL, Torres-Lagares D. Outcome of single immediate implants placed in post-extraction infected and non-infected sites, restored with cemented crowns: a 3-year prospective study. J Dent. 2014 Jun;42(6):645-52. [Medline: 24675527] [doi: 10.1016/j.jdent.2014.03.008]

19. Jung RE, Zaugg B, Philipp AO, Truninger TC, Siegenthaler DW, Hämmerle CH. A prospective, controlled clinical trial evaluating the clinical radiological and aesthetic outcome after 5 years of immediately placed implants in sockets exhibiting periapical pathology. Clin Oral Implants Res. 2013 Aug;24(8):839-46. [Medline: 22672584] [doi: $10.1111 / \mathrm{j} .1600-0501.2012 .02491 . x]$

20. Crespi R, Capparè $\mathrm{P}$, Gherlone E. Immediate loading of dental implants placed in periodontally infected and noninfected sites: a 4-year follow-up clinical study. J Periodontol. 2010 Aug;81(8):1140-6. [Medline: 20370421] [doi: 10.1902/jop.2010.090666]

21. Crespi R, Capparè P, Gherlone E. Fresh-socket implants in periapical infected sites in humans. J Periodontol. 2010 Mar;81(3):378-83. [Medline: 20192864] [doi: 10.1902/jop.2009.090505]

22. Hita-Iglesias C, Sánchez-Sánchez FJ, Montero J, Galindo-Moreno P, Mesa F, Martínez-Lara I, Sánchez-Fernández E. Immediate Implants Placed in Fresh Sockets Associated with Periapical Pathology: A Split-Mouth Design and Survival Evaluation after 1-Year Follow-Up. Clin Implant Dent Relat Res. 2016 Dec;18(6):1075-1083. [Medline: 26676203] [doi: $10.1111 /$ cid.12387]

23. Blus C, Szmukler-Moncler S, Khoury P, Orrù G. Immediate implants placed in infected and noninfected sites after atraumatic tooth extraction and placement with ultrasonic bone surgery. Clin Implant Dent Relat Res. 2015 Jan;17 Suppl 1:e287-97. [Medline: 23899163] [doi: 10.1111/cid.12126]

24. Bell CL, Diehl D, Bell BM, Bell RE. The immediate placement of dental implants into extraction sites with periapical lesions: a retrospective chart review. J Oral Maxillofac Surg. 2011 Jun;69(6):1623-7. [Medline: 21496987] [doi: 10.1016/j.joms.2011.01.022]

25. Fugazzotto PA. A retrospective analysis of implants immediately placed in sites with and without periapical pathology in sixty-four patients. J Periodontol. 2012 Feb;83(2):182-6. [Medline: 21627462] [doi: 10.1902/jop.2011.110016]

26. Zuffetti F, Capelli M, Galli F, Del Fabbro M, Testori T. Post-extraction implant placement into infected versus noninfected sites: A multicenter retrospective clinical study. Clin Implant Dent Relat Res. 2017 Oct;19(5):833-840. [Medline: 28744958] [doi: 10.1111/cid.12523]

27. Fugazzotto P. A retrospective analysis of immediately placed implants in 418 sites exhibiting periapical pathology: results and clinical considerations. Int J Oral Maxillofac Implants. 2012 Jan-Feb;27(1):194-202. [Medline: 22299097]

28. Truninger TC, Philipp AO, Siegenthaler DW, Roos M, Hämmerle CH, Jung RE. A prospective, controlled clinical trial evaluating the clinical and radiological outcome after 3 years of immediately placed implants in sockets exhibiting periapical pathology. Clin Oral Implants Res. 2011 Jan;22(1):20-7. Erratum in: Clin Oral Implants Res. 2011 Feb;22(2):235. [Medline: 20678132] [doi: 10.1111/j.1600-0501.2010.01973.x]

29. Hamed MS. Clinical study of osseointegration of immediate implant in dentoalveolar socket with and without periapical pathosis. Gulf Med J. 2012;1(S1):S80-S89. [URL: https://www.semanticscholar.org/paper/Clinical-study-ofosseointegration-of-immediate-in-Hamed/a0a49693471ad9d6ff6894ed7ad568b140a2288c]

30. Nandal S, Ghalaut P, Shekhawat H. A radiological evaluation of marginal bone around dental implants: An invivo study. Natl J Maxillofac Surg. 2014 Jul-Dec;5(2):126-37. [Medline: 25937721] [PMC free article: 4405952] [doi: $10.4103 / 0975-5950.154813$ ] 
31. Koutouzis T. Crestal Bone Level Alterations in Implant Therapy. In: Turkyilmaz I, editors. Implant Dentistry: A Rapidly Evolving Practice. London: IntechOpen; 2011. [doi: 10.5772/16477]

32. Albrektsson T, Isidor F. Consensus Report of Session IV. In: Lang NP, Karring T, editors. Proceedings of the First European Workshop on Periodontology. London: Quintessence Publishing; 1994. p. 365-9.

33. Berberi AN, Sabbagh JM, Aboushelib MN, Noujeim ZF, Salameh ZA. A 5-year comparison of marginal bone level following immediate loading of single-tooth implants placed in healed alveolar ridges and extraction sockets in the maxilla. Front Physiol. 2014 Jan 31;5:29. [Medline: 24550840] [PMC free article: 3908518] [doi: 10.3389/fphys.2014.00029]

34. Galindo-Moreno P, León-Cano A, Ortega-Oller I, Monje A, O Valle F, Catena A. Marginal bone loss as success criterion in implant dentistry: beyond 2 mm. Clin Oral Implants Res. 2015 Apr;26(4):e28-e34. [Medline: 24383987] [doi: 10.1111/clr.12324]

35. Javaid MA, Khurshid Z, Zafar MS, Najeeb S. Immediate Implants: Clinical Guidelines for Esthetic Outcomes. Dent J (Basel). 2016 Jun 13;4(2):21. [Medline: 29563463] [PMC free article: 5851264] [doi: 10.3390/dj4020021]

36. Basualdo J, Ivankovic M, Kuzmicic J, Fernández E. Atraumatic extraction and immediate implant placement into infected site with the "ice cream cone" technique and L-PRF: A Case Report. Rev Clin Periodoncia Implantol Rehabil Oral. 2018 Apr;11(1):43-46. [doi: 10.4067/S0719-01072018000100043]

37. Monje A, Blasi G. Significance of keratinized mucosa/gingiva on peri-implant and adjacent periodontal conditions in erratic maintenance compliers. J Periodontol. 2019 May;90(5):445-453. [Medline: 30461016] [doi: 10.1002/JPER.18-0471]

38. Lin GH, Chan HL, Wang HL. The significance of keratinized mucosa on implant health: a systematic review. J Periodontol. 2013 Dec;84(12):1755-67. [Medline: 23451989] [doi: 10.1902/jop.2013.120688]

39. Pranskunas M, Poskevicius L, Juodzbalys G, Kubilius R, Jimbo R. Influence of Peri-Implant Soft Tissue Condition and Plaque Accumulation on Peri-Implantitis: a Systematic Review. J Oral Maxillofac Res. 2016 Sep 9;7(3):e2. [Medline: 27833727] [PMC free article: 5100642] [doi: 10.5037/jomr.2016.7302]

40. Chiu YW, Lee SY, Lin YC, Lai YL. Significance of the width of keratinized mucosa on peri-implant health. J Chin Med Assoc. 2015 Jul;78(7):389-94. [Medline: 26122633] [doi: 10.1016/j.jcma.2015.05.001]

41. Longoni S, Tinto M, Pacifico C, Sartori M, Andreano A. Effect of Peri-implant Keratinized Tissue Width on Tissue Health and Stability: Systematic Review and Meta-analysis. Int J Oral Maxillofac Implants. 2019 Nov/Dec;34(6):1307-1317. [Medline: 31711074] [doi: 10.11607/jomi.7622]

42. Pigozzo MN, Rebelo da Costa T, Sesma N, Laganá DC. Immediate versus early loading of single dental implants: A systematic review and meta-analysis. J Prosthet Dent. 2018 Jul;120(1):25-34. [Medline: 29703670] [doi: 10.1016/j.prosdent.2017.12.006]

43. Gallucci GO, Hamilton A, Zhou W, Buser D, Chen S. Implant placement and loading protocols in partially edentulous patients: A systematic review. Clin Oral Implants Res. 2018 Oct;29 Suppl 16:106-134. [Medline: 30328194] [doi: $10.1111 / \mathrm{clr} .13276]$

44. McCracken MS, Chavali RV, Al-Naief NS, Eleazer PD. A residual granuloma in association with a dental implant. Implant Dent. 2012 Apr;21(2):87-90. [Medline: 22382756] [doi: 10.1097/ID.0b013e31824c2b52]

45. Villar CC, Huynh-Ba G, Mills MP, Cochran DL. Wound healing around dental implants. Endod Topics. 2011 Jan;25(1): 44-62. [doi: 10.1111/etp.12018]

46. Li J, Jansen JA, Walboomers XF, van den Beucken JJ. Mechanical aspects of dental implants and osseointegration: A narrative review. J Mech Behav Biomed Mater. 2020 Mar;103:103574. [Medline: 32090904] [doi: 10.1016/j.jmbbm.2019.103574]

47. Esfahrood ZR, Kadkhodazadeh M, Amid R, Rokn A. Is The Periapical lesion a Risk For Periimplantitis? (A review). J Dent (Tehran). 2012 Spring;9(2):162-73. [Medline: 23066482] [PMC free article: 3466771]

48. Crespi R, Capparé P, Crespi G, Lo Giudice G, Gastaldi G, Gherlone E. Immediate Implant Placement in Sockets with Asymptomatic Apical Periodontitis. Clin Implant Dent Relat Res. 2017 Feb;19(1):20-27. [Medline: 27126371] [doi: $10.1111 / \mathrm{cid} .12422]$

49. Flanagan D. Enterococcus faecalis and Dental Implants. J Oral Implantol. 2017 Feb;43(1):8-11. [Medline: 27700696] [doi: 10.1563/aaid-joi-D-16-00069]

50. Kusek ER. Immediate implant placement into infected sites: bacterial studies of the Hydroacoustic effects of the YSGG laser. J Oral Implantol. 2011 Mar;37 Spec No:205-11. [Medline: 20712437] [doi: 10.1563/AAID-JOI-D-10-00014]

51. Rodríguez Sánchez F, Arteagoitia I, Rodríguez Andrés C, Caiazzo A. Antibiotic prophylaxis habits in oral implant surgery among dentists in Italy: a cross-sectional survey. BMC Oral Health. 2019 Dec 2;19(1):265. [Medline: 31791306 ] [PMC free article: 6889412] [doi: 10.1186/s12903-019-0943-x]

52. Romandini M, De Tullio I, Congedi F, Kalemaj Z, D’Ambrosio M, Laforí A, Quaranta C, Buti J, Perfetti G. Antibiotic prophylaxis at dental implant placement: Which is the best protocol? A systematic review and network meta-analysis. J Clin Periodontol. 2019 Mar;46(3):382-395. [Medline: 30729548] [doi: 10.1111/jcpe.13080]

53. Lee J, Park D, Koo KT, Seol YJ, Lee YM. Comparison of immediate implant placement in infected and noninfected extraction sockets: a systematic review and meta-analysis. Acta Odontol Scand. 2018 Jul;76(5):338-345. [Medline: 29611763] [doi: 10.1080/00016357.2018.1453084] 
54. Chen H, Zhang G, Weigl P, Gu X. Immediate placement of dental implants into infected versus noninfected sites in the esthetic zone: A systematic review and meta-analysis. J Prosthet Dent. 2018 Nov;120(5):658-667. [Medline: 29961634] [doi: 10.1016/j.prosdent.2017.12.008]

55. Negm SAM. Implant success versus implant survival. Dentistry. 2016:6(2):359. [doi: 10.4172/2161-1122.1000359]

56. Moraschini V, Poubel LA, Ferreira VF, Barboza Edos S. Evaluation of survival and success rates of dental implants reported in longitudinal studies with a follow-up period of at least 10 years: a systematic review. Int J Oral Maxillofac Surg. 2015 Mar;44(3):377-88. [Medline: 25467739] [doi: 10.1016/j.ijom.2014.10.023]

57. Simonis P, Dufour T, Tenenbaum H. Long-term implant survival and success: a 10-16-year follow-up of non-submerged dental implants. Clin Oral Implants Res. 2010 Jul;21(7):772-7. [Medline: 20636731] [doi: 10.1111/j.1600-0501.2010.01912.x]

58. Bhekare A, Elghannam M, Somji SH, Florio S, Suzuki T. Case Selection Criteria for Predictable Immediate Implant Placement and Immediate Provisionalization. J Oral Biol. 2018 Apr; 5(1):6. [doi: 10.13188/2377-987X.1000039]

59. Juodzbalys G, Sakavicius D, Wang HL. Classification of extraction sockets based upon soft and hard tissue components. J Periodontol. 2008 Mar;79(3):413-24. [Medline: 18315423] [doi: 10.1902/jop.2008.070397]

\section{To cite this article:}

Saijeva A, Juodzbalys G.

Immediate Implant Placement in Non-Infected Sockets versus Infected Sockets: a Systematic Review and Meta-Analysis

J Oral Maxillofac Res 2020;11(2):e1

URL: http://www.ejomr.org/JOMR/archives/2020/2/e1/v11n2e1.pdf

doi: $10.5037 /$ jomr.2020.11201

Copyright (C) Saijeva A, Juodzbalys G. Published in the JOURNAL OF ORAL \& MAXILLOFACIAL RESEARCH (http://www.ejomr.org), 30 June 2020.

This is an open-access article, first published in the JOURNAL OF ORAL \& MAXILLOFACIAL RESEARCH, distributed under the terms of the Creative Commons Attribution-Noncommercial-No Derivative Works 3.0 Unported License, which permits unrestricted non-commercial use, distribution, and reproduction in any medium, provided the original work and is properly cited. The copyright, license information and link to the original publication on (http://www.ejomr.org) must be included. 